\title{
ON STABILITY AND CONTROLLABILITY FOR SEMIGROUP ACTIONS
}

\author{
Josiney A. Souza - Hélio V.M. Tozatti - Victor H.L. Rocha
}

\begin{abstract}
This paper deals with stability and controllability for semigroup actions by using the topological method of admissible family of open coverings. The main results state a relationship of stable sets and control sets. The classical notion of controllability relates to the Poisson stability. The concept of prolongational control set relates to the Lyapunov stability.
\end{abstract}

\section{Introduction}

The present paper studies stability and controllability for semigroup actions on topological spaces. We introduce the notions of Poisson stable set, nonwandering set, and Lagrange stable set, which extend respectively the concepts of Poisson stable point, nonwandering point, and Lagrange stable motion. These concepts of stability theory can be connected by means of limit sets. We also present an aspect of controllability by prolongations that is related to the nonwandering points. The so-called prolongational control set is the link between Lyapunov stability and controllability.

The concept of stable set for dynamical systems on metric spaces was extensively studied by Bhatia and Szegö [2] and [3]. Bhatia and Hajek [1] developed a theory of stability for local semidynamical systems on topological spaces. Recently, Braga Barros, Souza and Rocha [6] introduced a theory of Lyapunov

2010 Mathematics Subject Classification. Primary: 37B05, 37B25, 93D05, 93B05.

Key words and phrases. Semigroup action; stability theory; control theory.

Research supported by the Fundação Araucária grant no. 476/14 and CNPq grant no. 476024/2012-9 Universal 14/2012 
stability of sets for semigroup actions on topological spaces, extending several concepts and results of Lyapunov stable sets from [1]-[3]. On the other hand, the notion of controllability was extended from the setting of control systems to the setting of semigroup actions by San Martin [10]-[12]. Thus stability and controllability are both currently concepts of semigroup actions on topological spaces.

A questioning on the relationship of stability and controllability was incited in the paper [17], where a connection between the notions of Poisson stability and control set were stated, although the Poisson stability was named as the Poincaré recurrence. This connection was established by means of control sets depending on a family of subsets of the semigroup, which has stated an asymptotic aspect of controllability related to the well-known concept of topological transitivity. However, there is no study relating Lyapunov stability to controllability in the literature. This is due to the fact that the aspects of Lyapunov stability are totally different from the aspects of controllability. A Lyapunov stable set need not be controllable, while a control set need not be Lyapunov stable. The basic difference between these concepts is that the Lyapunov stability concerns the dynamics in each neighbourhood of a set, while the controllability considers an equivalence class by almost transitivity among the points inside the set. Our intention in the present paper is to explain a situation in what these concepts are connected.

Let $(S, M)$ be a semigroup action on the topological space $M$. A set $X \subset M$ is controllable if $X \subset \operatorname{cls}(S x)$ for every $x \in X$. On the other hand, if $X \subset M$ is equistable then $\mathrm{D}(x, S) \subset X$ for all $x \in X$, where $\mathrm{D}(x, S)$ is the forward prolongation of $x$, which implies $\operatorname{cls}(S x) \subset X$ for every $x \in X$. Thus controllable and equistable sets are technically distinct, although both concepts concern the orbits through the points of the set. Then we introduce two objects which approximate controllability and stability: the weak prolongational control set and the minimal equistable set. A set $X \subset M$ is minimal equistable if it is nonempty, closed, equistable, and has no proper subset satisfying these properties. A subset $E \subset M$ is weak controllable by prolongations if each point $y$ in $E$ can be reachable by prolongations from another point $x$ in $E$. In other words, $E$ is weak controllable by prolongations if $E \subset \mathrm{D}(x, S)$ for every $x \in E$. The set $E$ is called a weak prolongational control set if it is weak controllable by prolongations and is maximal satisfying this property. In general, if $\mathcal{F}$ is a family of subsets of the semigroup $S$, then $E$ is a weak prolongational $\mathcal{F}$-control set if $E \subset \mathrm{J}(x, \mathcal{F})$ for every $x \in E$, where $\mathrm{J}(x, \mathcal{F})$ is the forward $\mathcal{F}$-prolongational limit set of $x$, and $E$ is maximal with this property. The notions of nonwandering point and dispersiveness are involved in this study. A point $x \in M$ is $\mathcal{F}$-nonwandering if $x \in \mathrm{J}(x, \mathcal{F})$, while the action is dispersive if $\mathrm{J}(x, \mathcal{F})=\emptyset$ for every $x \in M$ (see [19]). Thus 
each point in a weak prolongational $\mathcal{F}$-control set is $\mathcal{F}$-nonwandering and every $\mathcal{F}$-nonwandering point is contained in a weak prolongational $\mathcal{F}$-control set. Nevertheless, if the action is dispersive, then there is no weak prolongational $\mathcal{F}$-control set. If the action is equicontinuous then the weak prolongational $\mathcal{F}$ control sets form a partition for the set of all $\mathcal{F}$-nonwandering points, since they are equivalence classes to an order relation among the $\mathcal{F}$-nonwandering points. The main result in such a situation says that a compact minimal equistable set is a weak prolongational $\mathcal{F}$-control set that is maximal with respect to a dynamic order among the weak prolongational $\mathcal{F}$-control sets, and the converse holds if the whole space is compact.

\section{Semigroup actions}

In this section, we give the standard notations of semigroup actions on topological spaces. We recall the definitions and properties of transitivity, limit sets, and prolongational limit sets.

We start with some standard notations of semigroup actions. An action (or a left action) of $S$ on $M$ is a mapping

$$
\mu: S \times M \rightarrow M, \quad(s, x) \mapsto \mu(s, x)=s x
$$

satisfying $s(u x)=(s u) x$ for all $x \in M$ and $u, s \in S$. In this case we say that $S$ acts on $M$. We denote by $\mu_{s}: M \rightarrow M$ the map defined by $\mu_{s}(x)=\mu(s, x)$. We assume that $\mu_{s}$ is continuous for every $s \in S$. The action $\mu$ is called open if $\mu_{s}$ is an open map for every $s \in S$. We will often indicate the action of $S$ on $M$ by the triple $(S, M, \mu)$, or simply $(S, X)$.

Let $(S, M, \mu)$ be a fixed semigroup action. For subsets $X \subset M$ and $A \subset S$ we define the sets

$$
\begin{gathered}
A X=\bigcup_{s \in A} \mu_{s}(X)=\{y \in M \text { : there exist } s \in A \text { and } x \in X \text { with } s x=y\}, \\
A^{*} X=\bigcup_{s \in A} \mu_{s}^{-1}(X)=\{y \in M \text { : there exist } s \in A \text { and } x \in X \text { with } s y=x t\} .
\end{gathered}
$$

The sets $S x$ and $S^{*} x$ are called respectively the orbit of $x$ and the backward orbit of $x$ in $M$.

A set $X \subset M$ is called forward invariant or backward invariant if respectively $S X \subset X$ or $S^{*} X \subset X$; it is called invariant if it is forward and backward invariant. The set $X$ is said to be a forward minimal set if $X$ is nonempty, closed, forward invariant, and $X$ has no proper subset with these properties. It is easily seen that the set $X$ is a forward minimal if and only if $\operatorname{cls}(S x)=X$, for every $x \in X$. A point $x \in M$ is said to be rest if $\{x\}=S x$; weak transitive if $x \in \operatorname{cls}(S x)$; transitive if $x \in S x$; almost periodic if $\operatorname{cls}(S x)$ is a forward minimal set containing $x$; and periodic if $S x$ is a forward minimal set containing $x$. 
Definition 2.1. Let $\mathcal{F}$ be a family of subsets of the semigroup $S$. The $\omega$-limit set of $X \subset M$ for the family $\mathcal{F}$ is defined by

$$
\omega(X, \mathcal{F})=\bigcap_{A \in \mathcal{F}} \operatorname{cls}(A X)
$$

and the $\omega^{*}$-limit set of $X \subset M$ for $\mathcal{F}$ by

$$
\omega^{*}(X, \mathcal{F})=\bigcap_{A \in \mathcal{F}} \operatorname{cls}\left(A^{*} X\right) .
$$

Note that the limit sets of $(S, X)$ with respect to the family $\mathcal{F}$ are nonempty if $X$ is a compact forward invariant subspace of $M$ and $\mathcal{F}$ is a filter basis on the subsets of $S$ (i.e. $\emptyset \notin \mathcal{F}$ and given $A, B \in \mathcal{F}$ there is $C \in \mathcal{F}$ with $C \subset A \cap B$ ). We might assume the following additional hypotheses on the family $\mathcal{F}$.

Definition 2.2. The family $\mathcal{F}$ is said to satisfy:

(a) Hypothesis $\left(\mathrm{H}_{1}\right)$ if for all $s \in S$ and $A \in \mathcal{F}$ there exists $B \in \mathcal{F}$ such that $s B \subset A$.

(b) Hypothesis $\left(\mathrm{H}_{2}\right)$ if for all $s \in S$ and $A \in \mathcal{F}$ there exists $B \in \mathcal{F}$ such that $B s \subset A$.

(c) Hypothesis $\left(\mathrm{H}_{3}\right)$ if for all $s \in S$ and $A \in \mathcal{F}$ there exists $B \in \mathcal{F}$ such that $B \subset A s$.

These hypotheses play an important role in the investigation of invariance issues. For instance, hypothesis $\left(\mathrm{H}_{1}\right)$ yields the limit set $\omega(X, \mathcal{F})$ is forward invariant; $\left(\mathrm{H}_{3}\right)$ guarantees that $\omega^{*}(X, \mathcal{F})$ is forward invariant; and $\left(\mathrm{H}_{2}\right)$ assures that $\omega^{*}(X, \mathcal{F})$ is backward invariant whenever the action is open (see [5, Propositions $2.10,2.12,2.13])$. Moreover, it is easily seen that $\omega(s x, \mathcal{F}) \subset \omega(x, \mathcal{F})$ for all $x \in M$ and $s \in S$ if $\mathcal{F}$ satisfies hypothesis $\left(\mathrm{H}_{2}\right)$, and $\omega(x, \mathcal{F}) \subset \omega(s x, \mathcal{F})$ for all $x \in M$ and $s \in S$ if $\mathcal{F}$ satisfies hypothesis $\left(\mathrm{H}_{3}\right)$. We remark that hypotheses $\left(\mathrm{H}_{1}\right),\left(\mathrm{H}_{2}\right)$, and $\left(\mathrm{H}_{3}\right)$ are satisfied in general cases, including the families which define limit sets for flows, semiflows, and certain class of control systems (see [5] and [13]).

Note that hypotheses $\left(\mathrm{H}_{1}\right)$ and $\left(\mathrm{H}_{2}\right)$ just extend the notion of ideals. Recall that a nonempty subset $I \subseteq S$ is a left ideal if it is closed and $S I \subseteq I$. Similarly, one defines a right ideal and a two-sided ideal. If $\mathcal{F}$ is a family of left ideals of $S$, then $s A \subset A$ for all $s \in S$ and $A \in \mathcal{F}$. Hence, $\mathcal{F}$ satisfies $\left(\mathrm{H}_{1}\right)$. If $\mathcal{F}$ is a family of right ideals of $S$, then $A s \subset A$ for all $s \in S$ and $A \in \mathcal{F}$. Hence, $\mathcal{F}$ satisfies $\left(\mathrm{H}_{2}\right)$. The family considered in the following examples consists of left ideals.

EXAMPLE 2.3. This example generalizes limit sets for flows and semiflows. Let $(S, M)$ be a semigroup action and consider the family of translates

$$
\mathcal{F}=\{S t: t \in S\}
$$


The elements of $\mathcal{F}$ are clearly left ideals of $S$. The direction for limit behavior established by the family $\mathcal{F}$ relates to the well-known Green's $\mathcal{L}$-preorder of semigroup theory. It is easily seen that the family $\mathcal{F}$ also satisfies hypothesis $\left(\mathrm{H}_{3}\right)$. Furthermore, if $S$ is right reversible $(S t \cap S s \neq \emptyset$ for all $t, s \in S)$, then $\mathcal{F}$ is also a filter basis on the subsets of $S$.

EXAMPLE 2.4. Consider the following affine control system:

$$
\begin{gathered}
\dot{x}(t)=X_{0}(x(t))+\sum_{i=1}^{n} u_{i}(t) X_{i}(x(t)), \\
u \in \mathcal{U}_{\mathrm{pc}}=\{u \in \mathcal{U}, u \text { piecewise constant }\}
\end{gathered}
$$

on a connected $d$-dimensional manifold $M$, where $U \subset \mathbb{R}^{n}$ is the control range and $X_{0}, \ldots, X_{n}$ are vector fields in $M$. For each $u \in \mathcal{U}$ and $x \in M$ we denote by $\varphi(t, x, u)$ the solution of the system at time $t \in \mathbb{R}$, with $\varphi(0, x, u)=x$. For each $t \in \mathbb{R}$ and $u \in \mathcal{U}$, one has the diffeomorphism $\varphi_{t}^{u}: M \rightarrow M$, where $\varphi_{t}^{u}(x)=\varphi(t, x, u)$ for all $x \in X$. For each $x \in M$ and $u=\left(u_{1}, \ldots, u_{n}\right) \in \mathbb{R}^{n}$, we define the vector field $X_{u}(x)=X_{0}(x)+\sum_{i=1}^{n} u_{i} X_{i}(x)$. Then the control system is determined by the corresponding set of vector fields $F=\left\{X_{u}: u \in U\right\}$. The system semigroup is the semigroup

$$
S=\left\{\mathrm{e}^{t_{n} Y_{n}} \mathrm{e}^{t_{n-1} Y_{n-1}} \ldots \mathrm{e}^{t_{1} Y_{1}}: Y_{j} \in F, t_{j} \geq 0, n \in \mathbb{N}\right\}
$$

which corresponds to the solutions for the system with the piecewise constant controls in $\mathcal{U}_{\mathrm{pc}}$. For each $t>0$, we define the subsemigroup $S_{\geq t}$ of $S$ as

$$
S_{\geq t}=\left\{\varphi_{s}^{u}: s \geq t \text { and } u \in \mathcal{U}_{\mathrm{pc}}\right\}
$$

The family $\mathcal{F}=\left\{S_{\geq t}: t>0\right\}$ is a time-dependent filter basis on the subsets of $S$, since $S_{\geq t+s} \subset S_{\geq t} \cap S_{\geq s}$ for all $t, s>0$. As $\varphi_{s}^{u} S_{\geq t} \cup S_{\geq t} \varphi_{s}^{u} \subset S_{\geq t+s} \subset S_{\geq t}$ for all $t, s>0$ and $u \in \mathcal{U}_{\mathrm{pc}}, \mathcal{F}$ is a family of two-sided ideals of $S$.

The concept of prolongational limit set depends on a fixed admissible family of open coverings of $M$. If $\mathcal{U}$ and $\mathcal{V}$ are coverings of $M$, we write $\mathcal{V} \leq \mathcal{U}$ if $\mathcal{V}$ is a refinement of $\mathcal{U}$. We write $\mathcal{V} \leq \mathcal{U} / 2$ if for every $V, V^{\prime} \in \mathcal{V}$, with $V \cap V^{\prime} \neq \emptyset$, there is $U \in \mathcal{U}$ such that $V \cup V^{\prime} \subset U$. For an open covering $\mathcal{U}$ of $M$ and a subset $X \subset M$, the $\mathcal{U}$-neighbourhood of $X$ is the set

$$
\begin{aligned}
\mathrm{B}(X, \mathcal{U}) & =\bigcup\{U \in \mathcal{U}: X \cap U \neq \emptyset\} \\
& =\{y \in M: \text { there are } x \in X \text { and } U \in \mathcal{U} \text { such that } x, y \in U\} .
\end{aligned}
$$

The $\mathcal{U}$-neighbourhood of $X$ is also called star of $X$ with respect to $\mathcal{U}$. We use the notation $\mathrm{B}(\{x\}, \mathcal{U})=\mathrm{B}(x, \mathcal{U})$ for $x \in M$. The concept of $\mathcal{U}$-neighbourhood for topological spaces generalizes the concept of $\varepsilon$-neighbourhood for metric spaces. 
Definition 2.5. A family $\mathcal{O}$ of open coverings of $M$ is said to be admissible if it satisfies the following properties:

(a) For each $\mathcal{U} \in \mathcal{O}$, there is $\mathcal{V} \in \mathcal{O}$ such that $\mathcal{V} \leq \mathcal{U} / 2$.

(b) If $X \subset M$ is an open set and $K$ is a compact set of $M$ contained in $X$, then there is an open covering $\mathcal{U} \in \mathcal{O}$ such that $\mathrm{B}(K, \mathcal{U}) \subset X$.

(c) For any $\mathcal{U}, \mathcal{V} \in \mathcal{O}$, there is $\mathcal{W} \in \mathcal{O}$ such that $\mathcal{W} \leq \mathcal{U}$ and $\mathcal{W} \leq \mathcal{V}$.

The space $M$ is called admissible if it has an admissible family of open coverings.

Note that an admissible family of open coverings is a direct set. Moreover, if $\mathcal{O}$ is an admissible family of open coverings of $M$, the collection of all $\mathcal{U}$ neighbourhoods $\mathrm{B}(x, \mathcal{U})$, with $x \in M$ and $\mathcal{U} \in \mathcal{O}$, is a basis for the topology of $M$. In general, every uniformizable space is admissible (see [14]). Thus, metric spaces, compact spaces, paracompact spaces, Tychonoff spaces, and topological groups are admissible spaces. The following result will be used afterwards.

Proposition 2.6. Let $\mathcal{O}$ be an admissible family of open coverings of $M$. Assume that $\left(x_{\mathcal{V}}\right)_{\mathcal{V} \in \mathcal{O}}$ and $\left(y_{\mathcal{V}}\right)_{\mathcal{V} \in \mathcal{O}}$ are two nets in $M$ satisfying $y_{\mathcal{V}} \in \mathrm{B}\left(x_{\mathcal{V}}, \mathcal{V}\right)$, for every $\mathcal{V} \in \mathcal{O}$. If $x_{\mathcal{V}} \rightarrow x$, then $y \mathcal{V} \rightarrow x$.

Proof. Let $\mathcal{U} \in \mathcal{O}$ be an open covering in $\mathcal{O}$ and take another open covering $\mathcal{W} \in \mathcal{O}$ such that $\mathcal{W} \leq \mathcal{U} / 2$. Since $x_{\mathcal{V}} \rightarrow x$ there is an open covering $\mathcal{V}_{0} \in \mathcal{O}$ such that $x_{\mathcal{V}} \in \mathrm{B}(x, \mathcal{W})$ whenever $\mathcal{V} \leq \mathcal{V}_{0}$. Now take an open covering $\mathcal{V}_{0}^{\prime} \in \mathcal{O}$ which refines both $\mathcal{V}_{0}$ and $\mathcal{W}$. For an open covering $\mathcal{V} \in \mathcal{O}$ which refines $\mathcal{V}_{0}^{\prime}$ one has $y_{\mathcal{V}} \in \mathrm{B}\left(x_{\mathcal{V}}, \mathcal{V}\right) \subset \mathrm{B}\left(x_{\mathcal{V}}, \mathcal{W}\right)$ and $x_{\mathcal{V}} \in \mathrm{B}(x, \mathcal{W})$. Thus there are open sets $W_{1}, W_{2} \in \mathcal{W}$ satisfying $y_{\mathcal{V}}, x_{\mathcal{V}} \in W_{1}$ and $x_{\mathcal{V}}, x \in W_{2}$. Choose an open set $U \in \mathcal{U}$ such that $W_{1} \cup W_{2} \subset U$. Then we have $y_{\mathcal{V}}, x \in U$, which means $y_{\mathcal{V}} \in \mathrm{B}(x, \mathcal{U})$. Therefore $y_{\mathcal{V}} \rightarrow x$.

From now on, and throughout the paper, there is a fixed filter basis $\mathcal{F}$ on the subsets of $S$ and an admissible family $\mathcal{O}$ of open coverings of $M$. The following definitions of prolongation and prolongational limit set were introduced in the setting of semigroup actions on metric spaces in [9] and in the setting of semigroup actions on general topological spaces in [6].

Definition 2.7. Let $x \in M$ and $A \subset S$. The first forward A-prolongation and the first backward $A$-prolongation of $x$ are defined respectively by

$$
\mathrm{D}(x, A)=\bigcap_{\mathcal{U} \in \mathcal{O}} \operatorname{cls}(A \mathrm{~B}(x, \mathcal{U})) \quad \text { and } \quad \mathrm{D}^{*}(x, A)=\bigcap_{\mathcal{U} \in \mathcal{O}} \operatorname{cls}\left(A^{*} \mathrm{~B}(x, \mathcal{U})\right) .
$$

For a given subset $X$ in $M$ we define $\mathrm{D}(X, A)=\bigcup_{x \in X} \mathrm{D}(x, A)$ and $\mathrm{D}^{*}(X, A)=$ $\bigcup_{x \in X} \mathrm{D}^{*}(x, A)$. Note that $\mathrm{D}(x, A)$ and $\mathrm{D}^{*}(x, A)$ are closed in $M, \operatorname{cl}(A x) \subset$ $\mathrm{D}(x, A)$, and $\operatorname{cl}\left(A^{*} x\right) \subset \mathrm{D}^{*}(x, A)$. If $K \subset M$ is compact, then $\mathrm{D}(K, A)$ is closed 
([6, Proposition 2.9]). Moreover, $\mathrm{D}(x, S)$ is forward invariant and, in the case of open action, $\mathrm{D}^{*}(x, S)$ is backward invariant.

Definition 2.8. The first forward $\mathcal{F}$-prolongational limit set and the first backward $\mathcal{F}$-prolongational limit set of $x \in M$ are defined respectively by

$$
\mathrm{J}(x, \mathcal{F})=\bigcap_{A \in \mathcal{F}} \mathrm{D}(x, A) \quad \text { and } \quad \mathrm{J}^{*}(x, \mathcal{F})=\bigcap_{A \in \mathcal{F}} \mathrm{D}^{*}(x, A) .
$$

For a given set $X \subset M$ we define $\mathrm{J}(X, \mathcal{F})=\bigcup_{x \in X} \mathrm{~J}(x, \mathcal{F})$ and $\mathrm{J}^{*}(X, \mathcal{F})=$ $\bigcup_{x \in X} \mathrm{~J}^{*}(x, \mathcal{F})$. It is easily seen that $\mathrm{J}(x, \mathcal{F})$ and $\mathrm{J}^{*}(x, \mathcal{F})$ are closed sets. Moreover, $\mathrm{J}(x, \mathcal{F})$ is forward invariant if $\mathcal{F}$ satisfies hypothesis $\left(\mathrm{H}_{1}\right), \mathrm{J}^{*}(x, \mathcal{F})$ is forward invariant if $\mathcal{F}$ satisfies hypothesis $\left(\mathrm{H}_{3}\right)$, and $\mathrm{J}^{*}(x, \mathcal{F})$ is backward invariant if $\mathcal{F}$ satisfies hypothesis $\left(\mathrm{H}_{2}\right)$ and the action is open ([6, Proposition 2.10]). Besides, one has $y \in \mathrm{J}(x, \mathcal{F})$ if and only if $x \in \mathrm{J}^{*}(y, \mathcal{F})$.

The following definition reproduces the notion of divergent net in the semigroup $S$.

Definition 2.9. For a given net $\left(t_{\lambda}\right)_{\lambda \in \Lambda}$ in $S$, the notation $t_{\lambda} \rightarrow_{\mathcal{F}} \infty$ means that for each $A \in \mathcal{F}$ there is $\lambda_{0} \in \Lambda$ such that $t_{\lambda} \in A$ for all $\lambda \geq \lambda_{0}$.

By considering the product direction on $\mathcal{F} \times \mathcal{O}$, that is, $(A, \mathcal{U}) \geq(B, \mathcal{V})$ if and only if $A \subset B$ and $\mathcal{U} \leq \mathcal{V}$, we can easily see that

$$
\omega(X, \mathcal{F})=\left\{\begin{array}{c}
x \in M: \text { there are nets }\left(t_{\lambda}\right)_{\lambda \in \Lambda} \text { in } S \text { and }\left(x_{\lambda}\right)_{\lambda \in \Lambda} \text { in } X \\
\text { such that } t_{\lambda} \rightarrow_{\mathcal{F}} \infty \text { and } t_{\lambda} x_{\lambda} \rightarrow x
\end{array}\right\},
$$

for any subset $X \subset M$, and

$$
\begin{aligned}
& \mathrm{D}(x, A)=\left\{\begin{array}{c}
y \in M: \text { there are nets }\left(t_{\lambda}\right) \text { in } A \text { and }\left(x_{\lambda}\right) \text { in } M \text { such that } \\
x_{\lambda} \rightarrow x \text { and } t_{\lambda} x_{\lambda} \rightarrow y
\end{array}\right\}, \\
& \mathrm{J}(x, \mathcal{F})=\left\{\begin{array}{c}
y \in M: \text { there are nets }\left(t_{\lambda}\right) \text { in } S \text { and }\left(x_{\lambda}\right) \text { in } M \text { such that } \\
t_{\lambda} \rightarrow \mathcal{F} \infty, x_{\lambda} \rightarrow x \text { and } t_{\lambda} x_{\lambda} \rightarrow y
\end{array}\right\},
\end{aligned}
$$

for any point $x \in M$.

We now define domains of attraction. They were introduced in [6] and [9], and play an important role in the studies of stability for compact sets.

Definition 2.10. Let $X \subset M$. The domain of weak $\mathcal{F}$-attraction of $X$ is the set

$$
\mathrm{A}_{w}(X, \mathcal{F})=\{x \in M: X \cap \omega(x, \mathcal{F}) \neq \emptyset\}
$$

the domain of $\mathcal{F}$-attraction of $X$ is the set

$$
\mathrm{A}(X, \mathcal{F})=\{x \in M: \omega(x, \mathcal{F}) \neq \emptyset \text { and } \omega(x, \mathcal{F}) \subset X\}
$$

the domain of weak uniform $\mathcal{F}$-attraction of $X$ is the set

$$
\mathrm{A}_{w u}(X, \mathcal{F})=\{x \in M: \mathrm{J}(x, \mathcal{F}) \cap X \neq \emptyset\}
$$


the domain of uniform $\mathcal{F}$-attraction of $X$ is the set

$$
\mathrm{A}_{u}(X, \mathcal{F})=\{x \in M: \mathrm{J}(x, \mathcal{F}) \neq \emptyset \text { and } \mathrm{J}(x, \mathcal{F}) \subset X\} .
$$

Any point $x$ in $\mathrm{A}_{w}(X, \mathcal{F}), \mathrm{A}(X, \mathcal{F}), \mathrm{A}_{w u}(X, \mathcal{F})$, or $\mathrm{A}_{u}(X, \mathcal{F})$ may respectively be said to be weakly $\mathcal{F}$-attracted, $\mathcal{F}$-attracted, weakly uniformly $\mathcal{F}$-attracted, or uniformly $\mathcal{F}$-attracted to $X$.

The following result characterizes the rest points by means of limit set and domain of attraction.

Proposition 2.11. Assume that the family $\mathcal{F}$ satisfies hypothesis $\left(\mathrm{H}_{1}\right)$. For $x \in M$, the following statements are equivalent:

(a) $x$ is a rest point.

(b) $\omega(x, \mathcal{F})=\{x\}$.

(c) $x \in \mathrm{A}(x, \mathcal{F})$.

Proof. The implications (a) $\Rightarrow(\mathrm{b}) \Rightarrow(\mathrm{c}) \Rightarrow(\mathrm{b})$ are immediate from the definitions. It remains to show that (b) implies (a). Indeed, since $\omega(x, \mathcal{F})$ is forward invariant, then $\omega(x, \mathcal{F})=\{x\}$ implies $S x \subset\{x\}$, and therefore $S x=\{x\}$.

\section{Stability}

We now deal with the stability theory for semigroup actions on topological spaces. We recall some definitions and properties of Lyapunov stability for sets and introduce the notions of Poisson and Lagrange stabilities for sets. We also discuss their connections. Throughout, there is a fixed semigroup action $(S, M)$, a family $\mathcal{F}$ of subsets of $S$, and an admissible family $\mathcal{O}$ of open coverings of $M$.

3.1. Lyapunov stability. We start defining some concepts of Lyapunov stability which are treated in this paper. We refer to [6] for definitions and properties of Lyapunov stability for semigroup actions on topological spaces.

Definition 3.1. A given set $X$ is said to be

(a) stable if for every $x \in X$ and every open covering $\mathcal{U} \in \mathcal{O}$ there exists $\mathcal{V} \in \mathcal{O}$ such that $S \mathrm{~B}(x, \mathcal{V}) \subset \mathrm{B}(X, \mathcal{U})$

(b) uniformly stable if for every open covering $\mathcal{U} \in \mathcal{O}$ there exists $\mathcal{V} \in \mathcal{O}$ such that $S \mathrm{~B}(X, \mathcal{V}) \subset \mathrm{B}(X, \mathcal{U})$;

(c) equistable if for each $x \notin X$ there exists $\mathcal{U} \in \mathcal{O}$ such that $x \notin \operatorname{cls}(S \mathrm{~B}(X, \mathcal{U}))$.

Note that a uniformly stable set is stable and a compact stable set is uniformly stable. Moreover, a closed uniformly stable set is equistable ([6, Theorem 3.3]), while an equistable or closed stable set is forward invariant ([6, Proposition 3.1]).

The next result presents a general property of equistable sets. 
Proposition 3.2. If $X \subset M$ is an equistable set, then $\mathrm{D}(x, S) \subset X$ for every $x \in X$.

Proof. Suppose that there is $x \in X$ such that $\mathrm{D}(x, S) \nsubseteq X$ and take $y \in \mathrm{D}(x, S) \backslash X$. Then there are nets $\left(t_{\lambda}\right)_{\lambda \in \Lambda}$ in $S$ and $\left(x_{\lambda}\right)_{\lambda \in \Lambda}$ in $M$ such that $x_{\lambda} \rightarrow x$ and $t_{\lambda} x_{\lambda} \rightarrow y$, and there is an open covering $\mathcal{U} \in \mathcal{O}$ satisfying $y \notin \operatorname{cls}(\mathcal{S B}(X, \mathcal{U}))$. By the convergence of $\left(x_{\lambda}\right)$, there is $\lambda_{0} \in \Lambda$ such that $x_{\lambda} \in \mathrm{B}(x, \mathcal{U})$ whenever $\lambda \geq \lambda_{0}$. Thus, we have $t_{\lambda} x_{\lambda} \in S \mathrm{~B}(x, \mathcal{U})$ whenever $\lambda \geq \lambda_{0}$. It follows that $y \in \operatorname{cls}(S \mathrm{~B}(x, \mathcal{U}))$, which is a contradiction.

In the case of compact set, we have the following theorem proved in $[6$, Corollary 3.1, Proposition 3.3].

THEOREM 3.3. Let $K \subset M$ be compact.

(a) If $\mathrm{D}(K, S)=K$, then $K$ is equistable. Conversely, if $K$ is equistable and every point in $K$ is weak transitive, then $\mathrm{D}(K, S)=K$.

(b) If $K$ is stable and every point in $K$ is weak transitive, then $\mathrm{D}(K, S)=K$. Conversely, if $\mathrm{D}(K, S)=K, M$ is locally compact, every point in $K$ is weak transitive, and every orbit of $S$ has connected closure, then $K$ is stable.

REMARK 3.4. The studies of Lyapunov stability for points reduce to the rest points. In fact, suppose that $x \in M$ is equistable. For all $s \in S$ and $\mathcal{U} \in \mathcal{O}$ we have $s x \in \operatorname{cls}(S \mathrm{~B}(x, \mathcal{U})$. As $\{x\}$ is equistable, it means that $s x \in\{x\}$, that is, $s x=x$.

3.2. Poisson stability. We now introduce the concept of Poisson stable set that is connected with recursiveness.

Definition 3.5. A given set $X \subset M$ is said to be forward $\mathcal{F}$-recursive with respect to the nonempty subset $Z \subset M$ if for every $A \in \mathcal{F}, A Z \cap X \neq \emptyset$; the set $X$ is said to be backward $\mathcal{F}$-recursive with respect to the set $Z$ if for every $A \in \mathcal{F}, A^{*} Z \cap X \neq \emptyset$.

Definition 3.6. A given set $X$ is said to be

(a) weakly forward $\mathcal{F}$-Poisson stable if every neighbourhood of $X$ is forward $\mathcal{F}$-recursive with respect to $X$,

(b) weakly backward $\mathcal{F}$-Poisson stable if every neighbourhood of $X$ is backward $\mathcal{F}$-recursive with respect to the set $X$,

(c) weakly $\mathcal{F}$-Poisson stable if $X$ is weakly forward and weakly backward $\mathcal{F}$-Poisson stable,

(d) forward $\mathcal{F}$-Poisson stable if every open set $U$ with $X \cap U \neq \emptyset$ is forward $\mathcal{F}$-recursive with respect to $X$, 
(e) backward $\mathcal{F}$-Poisson stable if every open set $U$ with $X \cap U \neq \emptyset$ is backward $\mathcal{F}$-recursive with respect to $X$,

(f) $\mathcal{F}$-Poisson stable if $X$ is forward and backward $\mathcal{F}$-Poisson stable.

Note that a forward $\mathcal{F}$-Poisson stable set is weakly forward $\mathcal{F}$-Poisson stable, and a backward $\mathcal{F}$-Poisson stable set is weakly backward $\mathcal{F}$-Poisson stable. In particular, an $\mathcal{F}$-Poisson stable set is weakly $\mathcal{F}$-Poisson stable.

The following theorem provides an alternative definition of weak Poisson stability for compact sets.

THEOREM 3.7. Let $X \subset M$ be a nonempty set.

(1) If $\omega(X, \mathcal{F}) \cap X \neq \emptyset$ then $X$ is weakly forward $\mathcal{F}$-Poisson stable.

(a) If $X$ is compact, then $X$ is weakly forward $\mathcal{F}$-Poisson stable if and only if $\omega(X, \mathcal{F}) \cap X \neq \emptyset$.

(b) If $\omega^{*}(X, \mathcal{F}) \cap X \neq \emptyset$ then $X$ is weakly backward $\mathcal{F}$-Poisson stable.

(c) If $X$ is compact, then $X$ is weakly backward $\mathcal{F}$-Poisson stable if and only if $\omega^{*}(X, \mathcal{F}) \cap X \neq \emptyset$.

Proof. Suppose that $\omega(X, \mathcal{F}) \cap X \neq \emptyset$ and take $x \in \omega(X, \mathcal{F}) \cap X$. Then $\mathrm{B}(x, \mathcal{U}) \cap A X \neq \emptyset$ for all $\mathcal{U} \in \mathcal{O}$ and $A \in \mathcal{F}$. As $\mathrm{B}(x, \mathcal{U}) \subset \mathrm{B}(X, \mathcal{U})$, it follows that every neighbourhood of $X$ is forward $\mathcal{F}$-recursive with respect to $X$. Thus, $X$ is weakly forward $\mathcal{F}$-Poisson stable, and item (a) is proved. Now, suppose that $X$ is compact and weakly forward $\mathcal{F}$-Poisson stable. Take an open neighbourhood $V$ of $X$. Then $V \cap A X \neq \emptyset$ for every $A \in \mathcal{F}$. Since $X$ is compact, there is $x \in X$ such that $x \in \operatorname{cls}(A X)$ for all $A \in \mathcal{F}$. Hence, $\omega(X, \mathcal{F}) \cap X \neq \emptyset$, and item (b) is proved. Items (c) and (d) are proved analogously to (a) and (b).

We now describe the principal aspect of the Poisson stability as follows.

THEOREM 3.8. For a given nonempty set $X$, the following statements are equivalent:

(a) $X$ is forward $\mathcal{F}$-Poisson stable.

(b) For each point $x \in X$ and $\mathcal{U} \in \mathcal{O}, \mathrm{B}(x, \mathcal{U})$ is forward $\mathcal{F}$-recursive with respect to $X$.

(c) $X \subset \omega(X, \mathcal{F})$.

Proof. First, we show that (a) implies (b). Suppose that $X$ is forward $\mathcal{F}$-Poisson stable, $x \in X$, and $\mathcal{U} \in \mathcal{O}$. Since $\mathrm{B}(x, \mathcal{U})$ is an open set such that $\mathrm{B}(x, \mathcal{U}) \cap X \neq \emptyset$, it follows that $\mathrm{B}(x, \mathcal{U})$ is forward $\mathcal{F}$-recursive with respect to $X$. Now, we show that (b) implies (c). Suppose that, for each $x \in X$ and $\mathcal{U} \in \mathcal{O}$, $\mathrm{B}(x, \mathcal{U})$ is forward $\mathcal{F}$-recursive with respect to $X$. Let $x \in X$ and $\mathcal{U} \in \mathcal{O}$. Then $\mathrm{B}(x, \mathcal{U}) \cap A X \neq \emptyset$ for all $A \in \mathcal{F}$. Hence, $x \in \operatorname{cls}(A X)$ for all $A \in \mathcal{F}$, that is, $x \in \omega(X, \mathcal{F})$. Thus, $X \subset \omega(X, \mathcal{F})$. Finally, we prove that (c) implies (a). 
Suppose that $X \subset \omega(X, \mathcal{F})$. If $U \subset M$ is an open set such that $X \cap U \neq \emptyset$, then $\omega(X, \mathcal{F}) \cap U \neq \emptyset$. It follows that $A X \cap U \neq \emptyset$ for all $A \in \mathcal{F}$, and therefore $U$ is forward $\mathcal{F}$-recursive with respect to $X$.

A similar theorem for backward Poisson stable sets is proved in the same way.

TheOREM 3.9. For a given nonempty set $X$, the following statements are equivalent:

(a) $X$ is backward $\mathcal{F}$-Poisson stable.

(b) For each point $x \in X$ and $\mathcal{U} \in \mathcal{O}, \mathrm{B}(x, \mathcal{U})$ is backward $\mathcal{F}$-recursive with respect to $X$.

(c) $X \subset \omega^{*}(X, \mathcal{F})$.

In particular, the set $X$ is $\mathcal{F}$-Poisson stable if and only if $X \subset \omega(X, \mathcal{F}) \cap$ $\omega^{*}(X, \mathcal{F})$. If the family $\mathcal{F}$ satisfies hypothesis $\left(\mathrm{H}_{1}\right)$ and $X$ is forward $\mathcal{F}$-Poisson stable, then $\operatorname{cls}(S X)=\omega(X, \mathcal{F})$. If the family $\mathcal{F}$ satisfies both hypotheses $\left(\mathrm{H}_{1}\right)$ and $\left(\mathrm{H}_{2}\right)$, and $X$ is $\mathcal{F}$-Poisson stable, then $\operatorname{cls}(S X)=\omega(X, \mathcal{F})=\omega^{*}(X, \mathcal{F})$.

Another consequence from Theorems 3.8 and 3.9 is that there is no difference between Poisson stability and weak Poisson stability for points. We also have the following corollaries from Theorems 3.8 and 3.9.

Corollary 3.10. Let $X \subset M$ be a nonempty set.

(a) If every point in $X$ is forward $\mathcal{F}$-Poisson stable, then $X$ is forward $\mathcal{F}$ Poisson stable.

(b) If every point in $X$ is backward $\mathcal{F}$-Poisson stable, then $X$ is backward $\mathcal{F}$-Poisson stable.

(c) If every point in $X$ is $\mathcal{F}$-Poisson stable, then $X$ is $\mathcal{F}$-Poisson stable.

Corollary 3.11. For $x \in X$, the following statements are equivalent:

(a) $x$ is forward $\mathcal{F}$-Poisson stable.

(b) $x \in \omega(x, \mathcal{F})$.

(c) $x \in \mathrm{A}_{w}(x)$.

Analogously, $x$ is backward $\mathcal{F}$-Poisson stable if and only if $x \in \omega^{*}(x, \mathcal{F})$, and $x$ is $\mathcal{F}$-Poisson stable if and only if $x \in \omega(x, \mathcal{F}) \cap \omega^{*}(x, \mathcal{F})$. Contrast to Corollary 3.11 with Proposition 2.11.

If $\mathcal{F}$ satisfies hypothesis $\left(\mathrm{H}_{1}\right)$, then $x$ is forward $\mathcal{F}$-Poisson stable if and only if $x$ is $\{S\}$-Poisson stable and $\operatorname{cls}(S x)=\omega(x, \mathcal{F})=\operatorname{cls}(A x)$, for every $A \in \mathcal{F}$. Moreover, if $\mathcal{F}$ also satisfies hypothesis $\left(\mathrm{H}_{3}\right)$, then the set of all forward $\mathcal{F}$-Poisson stable points is forward invariant (see [17]).

The next result relates invariance and Poisson stability.

Proposition 3.12. Let $X \subset M$ be a compact set. 
(a) If $X$ is forward invariant, then $X$ is weakly forward $\mathcal{F}$-Poisson stable.

(b) If $X$ is backward invariant, then $X$ is weakly backward $\mathcal{F}$-Poisson stable.

(c) If $X$ is invariant, then $X$ is $\mathcal{F}$-Poisson stable.

Proof. For item (a), suppose that $X$ is forward invariant. Then, for every $A \in \mathcal{F}$, we have $\operatorname{cls}(A X) \subset X$. As $\mathcal{F}$ is a filter basis and $X$ is compact, it follows that $\omega(X, \mathcal{F}) \neq \emptyset$ and $\omega(X, \mathcal{F}) \subset X$. By Theorem 3.7, $X$ is weakly forward $\mathcal{F}$-Poisson stable. Similarly, we prove item (b). For item (c), suppose that $X$ is invariant. Then $A X=A^{*} X=X$ for all $A \in \mathcal{F}$. Hence, $X=\omega(X, \mathcal{F})=$ $\omega^{*}(X, \mathcal{F})$. By Theorem 3.8, $X$ is $\mathcal{F}$-Poisson stable.

If $X$ is a closed Lyapunov stable set or an equistable set, then it is forward invariant. Then we have the following consequence from Proposition 3.12.

Corollary 3.13. If $X$ is a compact Lyapunov stable or equistable set, then it is weakly forward $\mathcal{F}$-Poisson stable.

Let $\mathcal{P}_{\mathcal{F}}$ denote the set of all forward $\mathcal{F}$-Poisson stable points

$$
\mathcal{P}_{\mathcal{F}}=\{x \in M: x \in \omega(x, \mathcal{F})\} .
$$

The closure of $\mathcal{P}_{\mathcal{F}}$ in $M$ is called the $\mathcal{F}$-Birkhoff center (or the set of central motions) of $(S, M)$. If $M$ is a separable metric space admitting a probability measure $\mu$ on the Borel sets of $M$ that is invariant under $S$, the Poincaré recurrence theorem says that $\mu$-almost every point in $M$ is forward $\mathcal{F}$-Poisson stable (see [17, Theorem 3.1]). In particular, if $\mu$ is a Radon measure such that $\mu(U)>0$ for any nonempty open set $U \subset X$, then $\mathcal{P}_{\mathcal{F}}$ is dense in $M$, that is, the $\mathcal{F}$-Birkhoff center of $(S, M)$ coincides with $M$.

We can define an equivalence relation in $\mathcal{P}_{\mathcal{F}}$ as follows:

$$
\text { for } x, y \in \mathcal{P}_{\mathcal{F}} \text {, let } x \sim_{\mathcal{F}} y \Leftrightarrow x \in \omega(y, \mathcal{F}) \text { and } y \in \omega(x, \mathcal{F}) \text {. }
$$

If $\mathcal{F}$ satisfies hypothesis $\left(\mathrm{H}_{1}\right)$, then this relation in $\mathcal{P}_{\mathcal{F}}$ is an equivalence relation (see $\left[17\right.$, p. 359]), and the equivalence classes of $\sim_{\mathcal{F}}$ coincide with the sets $P \subset M$ which satisfy the following properties:

(a) $P \subset \omega(x, \mathcal{F})$, for all $x \in P$,

(b) $P$ is maximal with property (a).

Note that $\omega(x, \mathcal{F})=\omega(y, \mathcal{F})$ for any points $x, y \in P$, that is, $P \subset \bar{P}$, where $\bar{P}=\omega(x, \mathcal{F})$ for all $x \in P$. In [17], each equivalence class of $\sim_{\mathcal{F}}$ was called a maximal $\mathcal{F}$-transitive set. By Theorem 3.8, the equivalence classes of the relation " $\sim \mathcal{F}$ " are $\mathcal{F}$-Poisson stable sets.

3.3. Nonwandering sets. We now define the concept of nonwandering set that is also connected with recursiveness and relates to the Poisson stability.

Definition 3.14. A given set $X$ is said to be 
(a) weakly $\mathcal{F}$-nonwandering if every neighbourhood $U$ of $X$ is forward $\mathcal{F}_{-}$ recursive with respect to itself, that is, $A U \cap U \neq \emptyset$ for all $A \in \mathcal{F}$,

(b) forward $\mathcal{F}$-nonwandering if every open set $V$ with $X \cap V \neq \emptyset$ is forward $\mathcal{F}$-recursive with respect to any neighbourhood $U$ of $X$,

(c) backward $\mathcal{F}$-nonwandering if every open set $V$ with $X \cap V \neq \emptyset$ is backward $\mathcal{F}$-recursive with respect to any neighbourhood $U$ of $X$,

(d) $\mathcal{F}$-nonwandering if it is forward and backward $\mathcal{F}$-nonwandering.

Note that a given set $X$ is weakly $\mathcal{F}$-nonwandering if and only if for every neighbourhood $U$ of $X$ and $A \in \mathcal{F}, A^{*} U \cap U \neq \emptyset$. This fact explains why we define neither weakly forward $\mathcal{F}$-nonwandering set nor weakly backward $\mathcal{F}$ nonwandering set. Note also that a forward or backward $\mathcal{F}$-nonwandering set is weakly $\mathcal{F}$-nonwandering.

We can describe the nonwandering sets by means of prolongational limit sets. We need the following property of prolongational limit sets.

Proposition 3.15. For $X \subset M, \mathrm{~J}(X, \mathcal{F}) \cap X \neq \emptyset$ if and only if $\mathrm{J}^{*}(X, \mathcal{F}) \cap$ $X \neq \emptyset$.

Proof. Suppose that $\mathrm{J}(X, \mathcal{F}) \cap X \neq \emptyset$ and take $x \in \mathrm{J}(X, \mathcal{F}) \cap X$. Then there is $y \in X$ such that $x \in \mathrm{J}(y, \mathcal{F})$. Hence, $y \in \mathrm{J}^{*}(x, \mathcal{F})$, and therefore $y \in \mathrm{J}^{*}(X, \mathcal{F}) \cap X$. The converse is proved in the same way.

Theorem 3.16. Let $X \subset M$ be a nonempty set. If $\mathrm{J}(X, \mathcal{F}) \cap X \neq \emptyset$ then $X$ is weakly $\mathcal{F}$-nonwandering. The converse holds if $X$ is compact.

Proof. Suppose that $\mathrm{J}(X, \mathcal{F}) \cap X \neq \emptyset$ and take $x \in \mathrm{J}(X, \mathcal{F}) \cap X$. Then there is $y \in X$ such that $x \in \mathrm{J}(y, \mathcal{F})$. Take an open neighbourhood $U$ of $X$ and $A \in \mathcal{F}$. There are nets $\left(t_{\lambda}\right)$ in $S$ and $\left(x_{\lambda}\right)$ in $M$ such that $t_{\lambda} \rightarrow_{\mathcal{F}} \infty, x_{\lambda} \rightarrow y$ and $t_{\lambda} x_{\lambda} \rightarrow x$. As $x, y \in U$, there is $\lambda_{0}$ such that $x_{\lambda}, t_{\lambda} x_{\lambda} \in U$ and $t_{\lambda} \in A$ for all $\lambda \geq \lambda_{0}$. Hence, $A U \cap U \neq \emptyset$, and therefore $X$ is $\mathcal{F}$-nonwandering. Now, suppose that $X$ is a compact weakly $\mathcal{F}$-nonwandering set. For $\mathcal{U} \in \mathcal{O}$ and $A \in \mathcal{F}$, we have $\mathrm{B}(X, \mathcal{U}) \cap A \mathrm{~B}(X, \mathcal{U}) \neq \emptyset$. Hence, there are $x_{(A, \mathcal{U})} \in \mathrm{B}(X, \mathcal{U})$ and $s_{(A, \mathcal{V})} \in A$ such that $s_{(A, \mathcal{V})} x_{(A, \mathcal{V})} \in \mathrm{B}(X, \mathcal{U})$. Since $X$ is compact, we may assume that the net $\left(x_{(A, \mathcal{U})}\right)$ converges to $x \in X$ and the net $\left(s_{(A, \mathcal{V})} x_{(A, \mathcal{V})}\right)$ converges to $y \in X$. As $s_{(A, \mathcal{V})} \rightarrow_{\mathcal{F}} \infty$, it follows that $y \in \mathrm{J}(x, \mathcal{F})$. Hence, $y \in \mathrm{J}(X, \mathcal{F}) \cap X$.

It is easily seen that a set $X$ is forward $\mathcal{F}$-nonwandering if for all $x \in X$ and $\mathcal{U} \in \mathcal{O}, \mathrm{B}(x, \mathcal{U})$ is forward $\mathcal{F}$-recursive with respect to any neighbourhood of $X$; $X$ is backward $\mathcal{F}$-nonwandering if for all $x \in X$ and $\mathcal{U} \in \mathcal{O}, \mathrm{B}(x, \mathcal{U})$ is backward $\mathcal{F}$-recursive with respect to any neighbourhood of $X$. The following theorem presents the main aspect of the nonwandering sets.

TheOREM 3.17. Let $X \subset M$ be a nonempty set. 
(a) If $X \subset \mathrm{J}(X, \mathcal{F})$, then $X$ is forward $\mathcal{F}$-nonwandering. The converse holds if $X$ is compact.

(b) If $X \subset \mathrm{J}^{*}(X, \mathcal{F})$, then $X$ is backward $\mathcal{F}$-nonwandering. The converse holds if $X$ is compact.

Proof. Suppose that $X \subset \mathrm{J}(X, \mathcal{F}), U$ is a neighbourhood of $X$, and $V$ is an open set such that $X \cap V \neq \emptyset$. Take $x \in X \cap V$ and $y \in X$ such that $x \in \mathrm{J}(y, \mathcal{F})$. Then $V \cap A \mathrm{~B}(y, \mathcal{U}) \neq \emptyset$ for all $A \in \mathcal{F}$ and $\mathcal{U} \in \mathcal{O}$. As $y \in U$, there is $\mathcal{U}$ such that $\mathrm{B}(y, \mathcal{U}) \subset U$. Hence, $V \cap A U \neq \emptyset$, and therefore $X$ is forward $\mathcal{F}$-nonwandering.

Now, suppose that $X$ is a compact forward $\mathcal{F}$-nonwandering set. Let $x \in X$, $A \in \mathcal{F}$, and $\mathcal{U} \in \mathcal{O}$. Then $A \mathrm{~B}(X, \mathcal{U}) \cap \mathrm{B}(x, \mathcal{U}) \neq \emptyset$. Take $x_{(A, \mathcal{U})} \in \mathrm{B}(X, \mathcal{U})$ and $s_{(A, \mathcal{V})} \in A$ such that $s_{(A, \mathcal{U})} x_{(A, \mathcal{U})} \in \mathrm{B}(x, \mathcal{U})$. Then $s_{(A, \mathcal{V})} \rightarrow_{\mathcal{F}} \infty$ and $s_{(A, \mathcal{U})} x_{(A, \mathcal{U})} \rightarrow x$. Moreover, $x_{(A, \mathcal{U})} \in \mathrm{B}\left(y_{(A, \mathcal{U})}, \mathcal{U}\right)$, with $y_{(A, \mathcal{U})} \in X$.

Since $X$ is compact, we may assume that the net $\left(y_{(A, \mathcal{U})}\right)$ converges to $y \in X$. Then the net $\left(x_{(A, \mathcal{U})}\right)$ also converges to $y$. In fact, for $\mathcal{V} \in \mathcal{O}$, take $\mathcal{V}^{\prime} \in \mathcal{O}$ such that $\mathcal{V}^{\prime} \leq \mathcal{V} / 2$ and take $\left(A_{0}, \mathcal{U}_{0}\right)$ such that $y_{(A, \mathcal{U})} \in \mathrm{B}\left(y, \mathcal{V}^{\prime}\right)$ for all $(A, \mathcal{U}) \geq$ $\left(A_{0}, \mathcal{U}_{0}\right)$. Choose $\mathcal{W} \in \mathcal{O}$ such that $\mathcal{W} \leq \mathcal{V}^{\prime}$ and $\mathcal{W} \leq \mathcal{U}_{0}$

For $(A, \mathcal{U}) \geq\left(A_{0}, \mathcal{W}\right)$, we have $y_{(A, \mathcal{U})} \in \mathrm{B}\left(y, \mathcal{V}^{\prime}\right)$ and $x_{(A, \mathcal{U})} \in \mathrm{B}\left(y_{(A, \mathcal{U})}, \mathcal{U}\right)$. Then there are $V^{\prime} \in \mathcal{V}^{\prime}$ and $U \in \mathcal{U}$ such that $y_{(A, \mathcal{U})}, y \in V^{\prime}$ and $y_{(A, \mathcal{U})}, x_{(A, \mathcal{U})} \in$ $U$. As $\mathcal{U} \leq \mathcal{W} \leq \mathcal{V}^{\prime}, \mathcal{V}^{\prime} \leq \mathcal{V} / 2$, and $y_{(A, \mathcal{U})} \in V^{\prime} \cap U$, there is $V \in \mathcal{V}$ such that $V^{\prime} \cup U \subset V$. Hence, $x_{(A, \mathcal{U})}, y \in V$, that is, $x_{(A, \mathcal{U})} \in \mathrm{B}(y, \mathcal{V})$. Thus, $x_{(A, \mathcal{U})} \rightarrow y$.

Finally, we have $s_{(A, \mathcal{V})} \rightarrow_{\mathcal{F}} \infty, x_{(A, \mathcal{U})} \rightarrow y$, and $s_{(A, \mathcal{U})} x_{(A, \mathcal{U})} \rightarrow x$, hence $x \in \mathrm{J}(y, \mathcal{F})$. Therefore, $X \subset \mathrm{J}(X, \mathcal{F})$, and item (a) is proved. Analogously, we prove item (b).

In particular, for $X$ compact, $X$ is $\mathcal{F}$-nonwandering if and only if $X \subset$ $\mathrm{J}(X, \mathcal{F}) \cap \mathrm{J}^{*}(X, \mathcal{F})$.

For points, there is no difference among the four items of Definition 3.14. The following results on nonwandering points are immediate consequences from Theorem 3.17.

Corollary 3.18. For $x \in X$, the following statements are equivalent:

(a) $x$ is $\mathcal{F}$-nonwandering.

(b) $x \in \mathrm{J}(x, \mathcal{F})$.

(c) For every neighbourhood $U$ of $x$ and $A \in \mathcal{F}, A^{*} U \cap U \neq \emptyset$.

(d) $x \in \mathrm{J}^{*}(x, \mathcal{F})$.

Corollary 3.19. Let $X \subset M$ be a nonempty set. If every point in $X$ is $\mathcal{F}$-nonwandering, then $X$ is $\mathcal{F}$-nonwandering.

In general, we have the following relations between Poisson stable sets and nonwandering sets: 
(1) A weakly forward (or backward) $\mathcal{F}$-Poisson stable set is weakly $\mathcal{F}$-nonwandering.

(2) A forward $\mathcal{F}$-Poisson stable set is forward $\mathcal{F}$-nonwandering.

(3) A backward $\mathcal{F}$-Poisson stable set is backward $\mathcal{F}$-nonwandering.

(4) An $\mathcal{F}$-Poisson stable set is $\mathcal{F}$-nonwandering.

For open sets, there is no difference between Poisson stable sets and nonwandering sets.

3.4. Lagrange stability. We finally define the notion of Lagrange stability for semigroup actions and present its connection with the Poisson stability.

Definition 3.20. A given set $X \subset M$ is said to be

(a) forward Lagrange stable if $\operatorname{cls}(S X)$ is compact,

(b) backward Lagrange stable if $\operatorname{cls}\left(S^{*} X\right)$ is compact,

(c) Lagrange stable if $\operatorname{cls}\left(S^{*} S X\right)$ is compact.

Proposition 3.21. If $X \subset M$ is Lagrange stable, then $\operatorname{cls}(S X) \subset \mathrm{A}(\operatorname{cls}(S X))$.

Proof. Let $x \in \operatorname{cls}(S X)$. Since $\operatorname{cls}(S X)$ is forward invariant, then $\operatorname{cls}(A x) \subset$ $\operatorname{cls}(S X)$ for all $A \in \mathcal{F}$. As $\mathcal{F}$ is a filter basis and $\operatorname{cls}(S X)$ is compact, it follows that $\omega(x, \mathcal{F}) \neq \emptyset$ and $\omega(x, \mathcal{F}) \subset \operatorname{cls}(S X)$. Hence, $x \in \mathrm{A}(\operatorname{cls}(S X))$.

The following result relates the Lagrange stability to the Poisson stability.

Proposition 3.22. If $X \subset M$ is forward Lagrange stable, then $\operatorname{cls}(S X)$ is a weakly forward $\mathcal{F}$-Poisson stable set.

Proof. Since $\operatorname{cls}(S X)$ is invariant and compact, the proof follows by Proposition 3.12 .

Besides, if $X \subset M$ is forward Lagrange stable, then there exists a forward minimal set $N \subset \operatorname{cls}(S X)$. If the family $\mathcal{F}$ satisfies hypothesis $\left(\mathrm{H}_{1}\right)$, it follows that $N=\omega(x, \mathcal{F})$ for all $x \in N$, and therefore every point in $N$ is forward $\mathcal{F}$-Poisson stable.

\section{Controllability}

In this section we introduce a relative control theory terminology. We refer to [4], [7], [10], and [12] for the control theory. Throughout, there is a fixed semigroup action $(S, M, \mu)$ and a filter basis $\mathcal{F}$ on the subsets of $S$.

Definition 4.1. A nonempty set $D \subset M$ is said to be a weak $\mathcal{F}$-control set if

(a) $D \subset \omega(x, \mathcal{F})$ for all $x \in D$,

(b) $D$ is maximal satisfying property (a). 
The set $D$ is called an $\mathcal{F}$-control set if it is a weak $\mathcal{F}$-control set with interior points.

This notion of controllability depending on a family of subsets of the semigroup introduces an asymptotic aspect to the control theory. Moreover, Definition 4.1 generalizes the original concept of control set. In fact, the notion of control set as stated in [10] coincides with the notion of $\{S\}$-control set, and the concept of weak control set as defined in [17] coincides with the concept of weak $\{S\}$-control set.

In general, a semigroup acting on a topological space is not transitive. Then the concept of control set was introduced with the intention of defining regions of the space where the transitivity occurs. Similarly, the $\mathcal{F}$-control sets define regions of the space where the asymptotic transitivity occurs. This property is related to the well-known concept of topological transitivity in dynamical systems and semigroup actions (see [16]).

Note that every weak $\mathcal{F}$-control set is an $\mathcal{F}$-Poisson stable set. Moreover, if $\mathcal{F}$ satisfies hypothesis $\left(\mathrm{H}_{1}\right)$, then the weak $\mathcal{F}$-control sets coincide with the equivalence classes of the equivalence relation " $\sim \mathcal{F}$ " in the set $\mathcal{P}_{\mathcal{F}}$ of all forward $\mathcal{F}$-Poisson stable points. Thus, weak $\mathcal{F}$-control sets are $\mathcal{F}$-Poisson stable. This is the relationship between the notions of Poisson stability and controllability. Nevertheless, the controllability is totally different from the Lyapunov stability. Let us see an elementary example that confirms this statement.

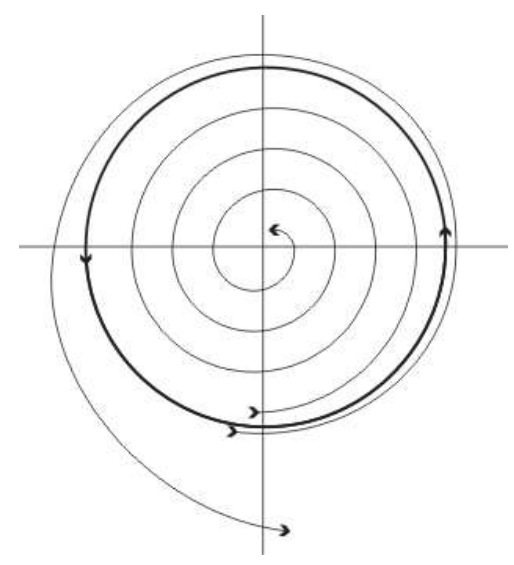

FIGURE 1. The unit circle is a weak control set that is Lyapunov unstable.

For time-reversal, the unit disk is Lyapunov stable but not controllable.

Example 4.2. Consider the dynamical system on $M=\mathbb{R}^{2}$ whose phase portrait is as in Figure 1. The origin 0 is a rest point and the unit circle $\mathbb{S}^{1}$ is a periodic trajectory. Hence, $\{0\}$ and $\mathbb{S}^{1}$ are two weak control sets (minimal 
sets). The trajectories in the interior of the unit circle spiral from the unit circle to the origin 0 as $t \rightarrow+\infty$. Hence, $\{0\}$ is Lyapunov stable. The trajectories in the exterior of $\mathbb{S}^{1}$ spiral away from $\mathbb{S}^{1}$. Thus, $\mathbb{S}^{1}$ is Lyapunov unstable. On the other hand, by considering the time-reversal dynamical system, the unit disk $\mathbb{D}^{1}$ is Lyapunov stable but not controllable.

Let $D \subset M$ be an $\mathcal{F}$-control set for $S$. The set

$$
D_{0}=\left\{x \in D: x \in \operatorname{int}(A x) \cap \operatorname{int}\left(A^{*} x\right) \text { for all } A \in \mathcal{F}\right\}
$$

is called the $\mathcal{F}$-transitivity set of $D$. If $D_{0}$ is nonempty, then the $\mathcal{F}$-control set $D$ is said to be an effective $\mathcal{F}$-control set.

REMARK 4.3. Let $A \subset S$ be a subsemigroup and $x \in M$. If $x \in \operatorname{int}\left(A^{*} x\right)$, then $A^{*} x$ is an open set. In fact, we have $A^{*} x \subset A^{*} \operatorname{int}\left(A^{*} x\right) \subset \operatorname{int}\left(A^{*} A^{*} x\right) \subset$ $\operatorname{int}\left(A^{*} x\right)$. Hence, $A^{*} x=\operatorname{int}\left(A^{*} x\right)$. In the same way, if the action of $A$ is open and $x \in \operatorname{int}(A x)$, then $A x$ is an open set.

The following result is proved in [17, Propositions 3.2 and 3.3].

Proposition 4.4. Assume that $\mathcal{F}$ is a family of subsemigroups of $S$. A nonempty subset $D \subset M$ is a weak $\mathcal{F}$-control set if and only if for each $A \in \mathcal{F}$ there is a weak control set $D_{A}$ of $A$ such that

$$
D=\bigcap_{A \in \mathcal{F}} D_{A} .
$$

Furthermore,

$$
\mathrm{A}_{w}(D)=\bigcap_{A \in \mathcal{F}} \mathrm{A}_{w}\left(D_{A}\right) .
$$

We have an additional result on transitivity sets, as follows.

Proposition 4.5. Assume that $\mathcal{F}$ is a family of subsemigroups of $S$. If $D \subset M$ is an $\mathcal{F}$-control set, then for each $A \in \mathcal{F}$ there is a control set $D_{A}$ of $A$ such that $D=\bigcap_{A \in \mathcal{F}} D_{A}$ and

$$
D_{0}=\bigcap_{A \in \mathcal{F}}\left(D_{A}\right)_{0}
$$

where $\left(D_{A}\right)_{0}$ is the transitivity set of $D_{A}$.

Proof. By Proposition 4.4, for each $A \in \mathcal{F}$ there is a weak control set $D_{A}$ of $A$ such that $D=\bigcap_{A \in \mathcal{F}} D_{A}$. Since $\operatorname{int}(D) \neq \emptyset$, we have $\operatorname{int}\left(D_{A}\right) \neq \emptyset$ for all $A \in \mathcal{F}$. Hence, $D_{A}$ is a control set of $A$, for each $A \in \mathcal{F}$. Let $x \in D_{0}$ and $A \in \mathcal{F}$. Then $x \in \operatorname{int}(A x) \cap \operatorname{int}\left(A^{*} x\right)$. Since $x \in D \subset D_{A}$, it follows that $x \in\left(D_{A}\right)_{0}$. As to the converse, if $x \in \bigcap_{A \in \mathcal{F}}\left(D_{A}\right)_{0}$, then $x \in D$ and $x \in \operatorname{int}(A x) \cap \operatorname{int}\left(A^{*} x\right)$, for all $A \in \mathcal{F}$. Thus $x \in D_{0}$. 
The next proposition extends a theorem that has been proved in the setting of semigroup of homeomorphisms, which states the properties of the transitivity sets of the effective control sets for a semigroup (see [4] and [12]).

Proposition 4.6. Let $A \subset S$ be a subsemigroup and $D \subset M$ an effective control set for $A$. The transitivity set $D_{0}$ has the following properties:

(a) $D \subset \operatorname{int}\left(A^{*} x\right)$ for every $x \in D_{0}$.

(b) $D_{0}=\operatorname{int}(A x) \cap \operatorname{int}\left(A^{*} x\right)$ for every $x \in D_{0}$.

(c) (total transitivity) For every $x, y \in D_{0}$ there is $s \in A$ such that $s x=y$.

(d) $D_{0}$ is dense in $D$.

(e) If the action of $A$ is open, then $D_{0}$ is $A$-invariant on $D$, that is, if $s \in A$, $x \in D_{0}$, and $s x \in D$, then $s x \in D_{0}$.

Proof. (a) Let $y \in D$ and $x \in D_{0}$. Then $x \in \operatorname{int}\left(A^{*} x\right)$ and $x \in \operatorname{cls}(A y)$. Hence, there is $s \in A$ such that $s y \in \operatorname{int}\left(A^{*} x\right)$. It follows that $y \in \mu_{s}^{-1}\left(\operatorname{int}\left(A^{*} x\right)\right)$ $\subset \operatorname{int}\left(A^{*} x\right)$. Thus $D \subset \operatorname{int}\left(A^{*} x\right)$.

(b) If $x \in D_{0}$ and $y \in \operatorname{int}(A x) \cap \operatorname{int}\left(A^{*} x\right)$, then there are $t, s \in A$ such that $y=t x$ and $x=s y$. Hence, $x \in \mu_{t}^{-1}(y)$, and therefore $y \in \operatorname{int}\left(A^{*} x\right) \subset$ $\operatorname{int}\left(A^{*} \mu_{t}^{-1}(x)\right) \subset \operatorname{int}\left(A^{*} y\right)$. Moreover, $y \in \operatorname{int}(A x)=\operatorname{int}(A s y) \subset \operatorname{int}(A y)$. Thus, $y \in \operatorname{int}(A y) \cap \operatorname{int}\left(A^{*} y\right)$. As $x \in A y$ and $y \in A x$, we have $y \in D$, and therefore $y \in D_{0}$. It follows that $\operatorname{int}(A x) \cap \operatorname{int}\left(A^{*} x\right) \subset D_{0}$ and $D_{0}$ is an open set. On the other hand, let $x, y \in D_{0}$. By item (a) we have $y \in \operatorname{int}\left(A^{*} x\right)$ and $x \in \operatorname{int}\left(A^{*} y\right)$. Hence, $y \in A x \cap \operatorname{int}\left(A^{*} x\right)$, and therefore $D_{0} \subset A x \cap \operatorname{int}\left(A^{*} x\right)$. Then we have $\operatorname{int}(A x) \cap \operatorname{int}\left(A^{*} x\right) \subset D_{0} \subset A x \cap \operatorname{int}\left(A^{*} x\right)$. Since $D_{0}$ is open, it follows that $D_{0}=\operatorname{int}(A x) \cap \operatorname{int}\left(A^{*} x\right)$.

(c) Follows immediately form item (b).

(d) Let $x \in D_{0}$. By item (a), we have $D \subset \operatorname{int}\left(A^{*} x\right)$. Since $D \subset \operatorname{cls}(A x)$, it follows that $D \subset \operatorname{cls}(A x) \cap \operatorname{int}\left(A^{*} x\right)$. By item (b), we have $\operatorname{cls}\left(D_{0}\right)=\operatorname{cls}(\operatorname{int}(A x) \cap$ $\left.\operatorname{int}\left(A^{*} x\right)\right)$. Hence

$$
D \subset \operatorname{cls}(A x) \cap \operatorname{int}\left(A^{*} x\right) \subset \operatorname{cls}\left(\operatorname{int}(A x) \cap \operatorname{int}\left(A^{*} x\right)\right)=\operatorname{cls}\left(D_{0}\right)
$$

and therefore $D_{0}$ is dense in $D$.

(e) Suppose that the action of $A$ is open, $s \in A, x \in D_{0}$, and $s x \in D$. By item (b) and Remark 4.3, we have $D_{0}=A x \cap A^{*} x$. By item (a), it follows that $s x \in D_{0}$.

By Remark 4.3, property (a) of Proposition 4.6 may be stated as $D \subset A^{*} x$ for every $x \in D_{0}$. If the action of $A$ is open, then property (b) may be enunciated as $D_{0}=A x \cap A^{*} x$ for every $x \in D_{0}$. As a consequence from Propositions 4.5 and 4.6, we have the following result on transitivity sets of control sets. 
Proposition 4.7. Assume that $\mathcal{F}$ is a family of subsemigroups of $S$. Let $D \subset$ $M$ be an effective $\mathcal{F}$-control set. The $\mathcal{F}$-transitivity set of $D$ has the following properties:

(a) $D \subset \omega^{*}(x, \mathcal{F})$ for every $x \in D_{0}$.

(b) $D_{0}=\bigcap_{A \in \mathcal{F}} \operatorname{int}\left(A x \cap A^{*} x\right)$ for all $x \in D_{0}$.

(c) For every $x, y \in D_{0}$ and $A \in \mathcal{F}$ there is $s_{A} \in A$ such that $s_{A} x=y$.

(d) If the action is open and $\mathcal{F}$ satisfies hypothesis $\left(\mathrm{H}_{1}\right)$, then $D_{0}$ is $S$ invariant on $D$, that is, if $s \in S, x \in D_{0}$, and $s x \in D$, then $s x \in D_{0}$.

Proof. Items (a)-(c) follow immediately from Propositions 4.5 and 4.6. For item (d), suppose that the action is open, $\mathcal{F}$ satisfies hypothesis $\left(\mathrm{H}_{1}\right), s \in S$, $x \in D_{0}$, and $s x \in D$. By item (a) of Proposition 4.6, we have $s x \in \bigcap_{A \in \mathcal{F}} \operatorname{int}\left(A^{*} x\right)$. For $A \in \mathcal{F}$, there is $B \in \mathcal{F}$ such that $s B \subset A$. As $x \in \operatorname{int}(B x)$, it follows that $s x \in \operatorname{sint}(B x) \subset \operatorname{int}(s B x) \subset \operatorname{int}(A x)$. Hence,

$$
s x \in \bigcap_{A \in \mathcal{F}} \operatorname{int}\left(A x \cap A^{*} x\right)=D_{0} .
$$

We now show that a set satisfying item (a) of Definition 4.1 may be extended to a weak $\mathcal{F}$-control set.

Proposition 4.8. Assume that $\mathcal{F}$ satisfies hypothesis $\left(\mathrm{H}_{1}\right)$. Assume that $D \subset M$ is a nonempty subset of $M$ satisfying $D \subset \omega(x, \mathcal{F})$ for all $x \in D$. Then there is a weak $\mathcal{F}$-control set $D^{\prime} \subset M$ containing $D$.

Proof. Let $\mathfrak{D}$ be the collection of sets

$$
\mathfrak{D}=\{C \subset M: D \subset C \text { and } C \subset \omega(x, \mathcal{F}) \text { for all } x \in C\} .
$$

Take an arbitrary chain $\left(C_{i}\right)_{i \in I}$ in $\mathfrak{D}$ and let $U=\bigcup_{i \in I} C_{i}$. Given $x, y \in U$, choose $j, k \in I$ such that $x \in C_{j}$ and $y \in C_{k}$ and fix $z \in D$. Then we have $z \in \omega(x, \mathcal{F})$ and $y \in \omega(z, \mathcal{F})$. It follows that $y \in \omega(x, \mathcal{F})$. Hence, $U \subset \omega(x, \mathcal{F})$ for all $x \in U$, and therefore $U \in \mathfrak{D}$. By Zorn's Lemma, there is a maximal set (with respect to the inclusion) $D^{\prime} \subset M$ such that $D^{\prime} \subset \omega(x, \mathcal{F})$ for all $x \in D^{\prime}$, and the result is proved.

This result shows that every forward $\mathcal{F}$-Poisson stable point is contained in a weak $\mathcal{F}$-control set whenever hypothesis $\left(\mathrm{H}_{1}\right)$ holds.

Corollary 4.9. Assume that $\mathcal{F}$ satisfies hypothesis $\left(\mathrm{H}_{1}\right)$. Assume that $D \subset M$ is a nonempty subset of $M$ satisfying:

(a) $\operatorname{int}(D) \neq \emptyset$ and

(b) $D \subset \omega(x, \mathcal{F})$ for all $x \in D$.

There is an $\mathcal{F}$-control set $D^{\prime} \subset M$ containing $D$. 
Proof. By Proposition 4.8, there is a weak $\mathcal{F}$-control set $D^{\prime}$ containing $D$. Since $\operatorname{int}(D) \neq \emptyset$ and $\operatorname{int}(D) \subset D^{\prime}$, the set $D^{\prime}$ is indeed an $\mathcal{F}$-control set.

A dynamic order among the weak $\mathcal{F}$-control sets of $(S, M)$ can be defined in the following way:

$\left(R_{\mathrm{CS}}\right)$ for two weak $\mathcal{F}$-control sets $D$ and $D^{\prime}$, let $D \preccurlyeq D^{\prime} \Leftrightarrow D \cap \mathrm{A}_{w}\left(D^{\prime}\right) \neq \emptyset$.

Proposition 4.10. Assume that $\mathcal{F}$ satisfies hypothesis $\left(\mathrm{H}_{1}\right)$. Then the relation $\left(R_{\mathrm{CS}}\right)$ among the weak $\mathcal{F}$-control sets is an order relation.

Proof. If $D$ is a weak $\mathcal{F}$-control set, then $D \subset \omega(x, \mathcal{F})$ for all $x \in D$. Hence, $D \subset \mathrm{A}_{w}(D)$ and therefore $D \preccurlyeq D$. If $D$ and $D^{\prime}$ are two weak $\mathcal{F}$-control sets such that $D \preccurlyeq D^{\prime}$ and $D^{\prime} \preccurlyeq D$, then $D \cap \mathrm{A}_{w}\left(D^{\prime}\right) \neq \emptyset$ and $D^{\prime} \cap \mathrm{A}_{w}(D) \neq \emptyset$. Take $x \in D \cap \mathrm{A}_{w}\left(D^{\prime}\right)$ and $y \in D^{\prime} \cap \mathrm{A}_{w}(D)$. Then $\omega(x, \mathcal{F}) \cap D^{\prime} \neq \emptyset$ and $\omega(y, \mathcal{F}) \cap D \neq \emptyset$. Now, take $u \in \omega(x, \mathcal{F}) \cap D^{\prime}$ and $v \in \omega(y, \mathcal{F}) \cap D$. As $x \in D$ and $y \in D^{\prime}$, we have $x \in \omega(v, \mathcal{F}) \subset \omega(y, \mathcal{F})$ and $y \in \omega(u, \mathcal{F}) \subset \omega(x, \mathcal{F})$. Hence, $x \sim_{\mathcal{F}} y$, and therefore $D=D^{\prime}$. Finally, suppose that $D \preccurlyeq D^{\prime}$ and $D^{\prime} \preccurlyeq D^{\prime \prime}$. Then $D \cap \mathrm{A}_{w}\left(D^{\prime}\right) \neq \emptyset$ and $D^{\prime} \cap \mathrm{A}_{w}\left(D^{\prime \prime}\right) \neq \emptyset$. Take $x \in D \cap \mathrm{A}_{w}\left(D^{\prime}\right)$ and $y \in D^{\prime} \cap \mathrm{A}_{w}\left(D^{\prime \prime}\right)$. Then there are $u \in \omega(x, \mathcal{F}) \cap D^{\prime}$ and $v \in \omega(y, \mathcal{F}) \cap D^{\prime \prime}$. As $y \in D^{\prime}$, it follows that $y \in \omega(u, \mathcal{F}) \subset$ $\omega(x, \mathcal{F})$, and $v \in \omega(y, \mathcal{F}) \subset \omega(u, \mathcal{F}) \subset \omega(x, \mathcal{F})$. Hence, $v \in \omega(x, \mathcal{F}) \cap D^{\prime \prime}$, and therefore $D \preccurlyeq D^{\prime \prime}$.

By using the dynamic order relation among the weak $\mathcal{F}$-control sets, we can present an alternative characterization of minimal subset in compact space, as follows.

Proposition 4.11. Assume that $M$ is a compact Hausdorff space and $\mathcal{F}$ satisfies hypotheses $\left(\mathrm{H}_{1}\right)$ and $\left(\mathrm{H}_{3}\right)$. Let $X \subset M$ be a nonempty subset. Then the following statements are equivalent:

(a) $X$ is a forward minimal set.

(b) $X$ is a closed weak $\mathcal{F}$-control set.

(c) $X$ is a weak $\mathcal{F}$-control set that is maximal with respect to the order relation $\left(R_{\mathrm{CS}}\right)$.

ProOF. See proof in [17], Proposition 3.4.

4.1. Prolongational control sets. We now introduce the notion of prolongational controllability for semigroup actions.

Definition 4.12. A nonempty set $E \subset M$ is said to be a weak prolongational $\mathcal{F}$-control set if

(a) $E \subset \mathrm{J}(x, \mathcal{F})$ for all $x \in E$,

(b) $E$ is maximal satisfying property (a). 
The set $E$ is called a prolongational $\mathcal{F}$-control set if it is a weak prolongational $\mathcal{F}$-control set with interior points.

If $A \subset S$ is a subsemigroup and $E \subset M$, we say that $E$ is a weak prolongational control set for $A$ if it is a weak prolongational $\{A\}$-control set. Thus, $E$ is a weak prolongational control set for $A$ if

(a) $E \subset \mathrm{D}(x, A)$ for all $x \in E$,

(b) $E$ is maximal satisfying property (a).

By Theorem 3.17, every weak prolongational $\mathcal{F}$-control set is an $\mathcal{F}$-nonwandering set.

Definition 4.13. Let $y \in M$. The action of $S$ on $M$ is said to be equicontinuous at $y$ if the family of functions $\left\{\mu_{s}\right\}_{s \in S}$ is equicontinuous at $y$, that is, for a given $\mathcal{U} \in \mathcal{O}$ there is $\mathcal{V} \in \mathcal{O}$ such that $s \mathrm{~B}(y, \mathcal{V}) \subset \mathrm{B}(s y, \mathcal{U})$ for all $s \in S$. The action is equicontinuous provide it is equicontinuous at every point in $M$.

Under equicontinuity we may describe the weak prolongational $\mathcal{F}$-control sets as equivalence classes for certain equivalence relation among the $\mathcal{F}$-nonwandering points. We need the following lemma.

Lemma 4.14. Assume that the action is equicontinuous at $y \in M$. If $x \in$ $\mathrm{D}(y, A)$ and $y \in \mathrm{D}(z, B)$, then $x \in \mathrm{D}(z, C)$, where $C \supset A B$.

Proof. Since $x \in \mathrm{D}(y, A)$, there are nets $\left(t_{\lambda}\right)$ in $A$ and $\left(y_{\lambda}\right)$ in $M$ such that $y_{\lambda} \rightarrow y$ and $t_{\lambda} y_{\lambda} \rightarrow x$. As $y \in \mathrm{D}(y, A)$, there are nets $\left(s_{\alpha}\right)$ in $B$ and $\left(z_{\alpha}\right)$ in $M$ such that $z_{\alpha} \rightarrow z$ and $s_{\alpha} z_{\alpha} \rightarrow y$. Let $\mathcal{U}, \mathcal{V} \in \mathcal{O}$ and take $\mathcal{W} \in \mathcal{O}$ with $\mathcal{W} \leq \mathcal{U} / 2$. By the equicontinuity at $y$, we can take $\mathcal{W}^{\prime} \in \mathcal{O}$ such that $s \mathrm{~B}\left(y, \mathcal{W}^{\prime}\right) \subset \mathrm{B}(s y, \mathcal{W})$ for all $s \in S$. There are indexes $\lambda_{0}$ and $\alpha_{0}$ such that $y_{\lambda}, s_{\alpha} z_{\alpha} \in \mathrm{B}\left(y, \mathcal{W}^{\prime}\right), t_{\lambda} y_{\lambda} \in \mathrm{B}(x, \mathcal{W})$, and $z_{\alpha} \in \mathrm{B}(z, \mathcal{V})$ for all $\lambda \geq \lambda_{0}$ and $\alpha \geq \alpha_{0}$. Hence, $t_{\lambda} y_{\lambda}, t_{\lambda} s_{\alpha} z_{\alpha} \in \mathrm{B}\left(t_{\lambda} y, \mathcal{W}\right)$ for all $\lambda \geq \lambda_{0}$ and $\alpha \geq \alpha_{0}$. As $\mathcal{W} \leq \mathcal{U} / 2$, it follows that $t_{\lambda} s_{\alpha} z_{\alpha} \in \mathrm{B}(x, \mathcal{U})$ with $t_{\lambda} s_{\alpha} \in A B$ and $z_{\alpha} \in \mathrm{B}(z, \mathcal{V})$. Hence, $\mathrm{B}(x, \mathcal{U}) \cap C \mathrm{~B}(z, \mathcal{V}) \neq \emptyset$, and therefore $x \in \mathrm{D}(z, C)$.

Now, assume that $\mathcal{F}$ is a family of subsemigroups of $S$ and denote by $\mathcal{N}_{\mathcal{F}}$ the set of all $\mathcal{F}$-nonwandering points

$$
\mathcal{N}_{\mathcal{F}}=\{x \in M: x \in \mathrm{J}(x, \mathcal{F})\} .
$$

We can define an equivalence relation in $\mathcal{N}_{\mathcal{F}}$ as follows:

$$
\text { for } x, y \in \mathcal{N}_{\mathcal{F}} \text {, let } x \approx_{\mathcal{F}} y \Leftrightarrow x \in \mathrm{J}(y, \mathcal{F}) \text { and } y \in \mathrm{J}(x, \mathcal{F}) \text {. }
$$

Assume that the action is equicontinuous. By Lemma 4.14, if $A \in \mathcal{F}, x \in$ $\mathrm{D}(y, A)$, and $y \in \mathrm{D}(z, A)$, then $x \in \mathrm{D}(z, A)$. Hence, $x \in \mathrm{J}(y, \mathcal{F})$ and $y \in \mathrm{J}(z, \mathcal{F})$ implies $x \in \mathrm{J}(z, \mathcal{F})$. Thus, the relation " $\approx_{\mathcal{F}}$ " is transitive. Since " $\approx_{\mathcal{F}}$ " is clearly reflexive and symmetric, it is an equivalence relation. It is not difficult to see 
that the equivalence classes of " $\approx \mathcal{F}$ " coincide with the weak prolongational $\mathcal{F}$ control sets. Hence, if $E$ is a weak prolongational $\mathcal{F}$-control set, then $E \subset \bar{E}$ where $\bar{E}=\mathrm{J}(x, \mathcal{F})$ for every $x \in E$.

From now on, we assume that the relation " $\approx \mathcal{F}$ " is an equivalence relation among the $\mathcal{F}$-nonwandering points. The next proposition shows that a set satisfying item (a) of Definition 4.12 can be extended to a weak prolongational $\mathcal{F}$-control set.

Proposition 4.15. Assume that $E \subset M$ is a nonempty subset satisfying $D \subset \mathrm{J}(x, \mathcal{F})$ for all $x \in E$. Then there is a weak prolongational $\mathcal{F}$-control set $E^{\prime}$ containing $E$.

Proof. The proof follows similarly to the proof of Proposition 4.8.

This result shows that every $\mathcal{F}$-nonwandering point is contained in a weak prolongational $\mathcal{F}$-control set. The next result is an immediate consequence from Proposition 4.15 .

Corollary 4.16. Assume that $E \subset M$ is a nonempty subset satisfying

(a) $\operatorname{int}(E) \neq \emptyset$ and

(b) $E \subset \mathrm{J}(x, \mathcal{F})$ for all $x \in E$.

Then there is a prolongational $\mathcal{F}$-control set $E^{\prime}$ containing $E$.

The next result describes a weak prolongational $\mathcal{F}$-control set as the intersection of weak prolongational control sets for the subsemigroups in $\mathcal{F}$.

Proposition 4.17. Assume that $\mathcal{F}$ is a family of subsemigroups of $S$. A nonempty subset $E \subset M$ is a weak prolongational $\mathcal{F}$-control set if and only if for each $A \in \mathcal{F}$ there is a weak prolongational control set $E_{A}$ for $A$ such that

$$
E=\bigcap_{A \in \mathcal{F}} E_{A} .
$$

Furthermore, if the action is equicontinuous, then

$$
\mathrm{A}_{w u}(E, \mathcal{F})=\bigcap_{A \in \mathcal{F}} \mathrm{A}_{w u}\left(E_{A}, A\right) .
$$

Proof. Let $E$ be a weak prolongational $\mathcal{F}$-control set. For each $A \in \mathcal{F}$, we have $E \subset \mathrm{J}(x, \mathcal{F}) \subset \mathrm{D}(x, A)$ for all $A \in \mathcal{F}$. Then there is a weak prolongational control set $E_{A}$ for $A$ such that $E \subset E_{A}$. Hence, $E \subset \bigcap_{A \in \mathcal{F}} E_{A}$. On the other hand, for any $x, y \in \bigcap_{A \in \mathcal{F}} E_{A}$, we have $y \in \bigcap_{A \in \mathcal{F}} E_{A} \subset \bigcap_{A \in \mathcal{F}} \mathrm{D}(x, A)=\mathrm{J}(x, \mathcal{F})$. Hence, the points of $\bigcap_{A \in \mathcal{F}}^{A} E_{A}$ lie in the same equivalent class of $\approx_{\mathcal{F}}$, and therefore $E=\bigcap_{A \in \mathcal{F}} E_{A}$. As to the converse, suppose that $E=\bigcap_{A \in \mathcal{F}} E_{A}$, where each $E_{A}$ is a weak prolongational control set for $A \in \mathcal{F}$. For $x \in E$, we have $E \subset \mathrm{J}(x, \mathcal{F})$. 
Hence, there is a weak prolongational $\mathcal{F}$-control set $E^{\prime}$ such that $E \subset E^{\prime}$. By the first part of this proof, it follows that $E^{\prime}=\bigcap_{A \in \mathcal{F}} E_{A}^{\prime}$, where each $E_{A}^{\prime}$ is a weak prolongational control set for $A \in \mathcal{F}$. Since distinct weak prolongational control sets are disjoint, we have $E_{A}=E_{A}^{\prime}$, for all $A \in \mathcal{F}$. Therefore $E=E^{\prime}$.

Now assume that $E=\bigcap_{A \in \mathcal{F}} E_{A}$ and take $x \in \mathrm{A}_{w u}(E, \mathcal{F})$. Then $\mathrm{J}(x, \mathcal{F}) \cap E \neq \emptyset$. It follows that $\mathrm{D}(x, A) \cap E_{A} \neq \emptyset$ for all $A \in \mathcal{F}$. Hence, $x \in \bigcap_{A \in \mathcal{F}} \mathrm{A}_{w u}\left(E_{A}, A\right)$. On the other hand, take $y \in \bigcap_{A \in \mathcal{F}} \mathrm{A}_{w u}\left(E_{A}, A\right)$. Then $\mathrm{D}(y, A) \cap E_{A} \neq \emptyset$ for all $A \in \mathcal{F}$. For each $A \in \mathcal{F}$, take $x_{A} \in \mathrm{D}(y, A) \cap E_{A}$. If $z \in E$ then $z \in \mathrm{D}\left(x_{A}, A\right)$ for all $A \in \mathcal{F}$. By Lemma 4.14, $z \in \mathrm{D}(y, A) \cap E$ for all $A \in \mathcal{F}$, that is, $z \in \mathrm{J}(y, \mathcal{F}) \cap E$. Therefore, $y \in \mathrm{A}_{w u}(E, \mathcal{F})$.

We now define the following relation among the weak prolongational $\mathcal{F}$ control sets of $(S, M)$ :

$\left(R_{\mathrm{PCS}}\right)$ for two weak prolongational $\mathcal{F}$-control sets $E$ and $E^{\prime}$,

$$
\text { let } E \preccurlyeq E^{\prime} \Leftrightarrow E \cap \mathrm{A}_{w u}\left(E^{\prime}\right) \neq \emptyset \text {. }
$$

Proposition 4.18. The relation $\left(R_{\mathrm{PCS}}\right)$ among the weak prolongational $\mathcal{F}$ control sets is an order relation.

Proof. If $E$ is a weak prolongational $\mathcal{F}$-control set, then $E \subset \mathrm{J}(x, \mathcal{F})$ for all $x \in E$. Hence, $E \subset \mathrm{A}_{w u}(E)$ and therefore $E \preccurlyeq E$. If $E$ and $E^{\prime}$ are two weak prolongational $\mathcal{F}$-control sets such that $E \preccurlyeq E^{\prime}$ and $E^{\prime} \preccurlyeq E$, then there are $x \in E \cap \mathrm{A}_{w u}\left(E^{\prime}\right)$ and $y \in E^{\prime} \cap \mathrm{A}_{w}(E)$, that is, $\mathrm{J}(x, \mathcal{F}) \cap E^{\prime} \neq \emptyset$ and $\mathrm{J}(y, \mathcal{F}) \cap E \neq \emptyset$. Take $u \in \mathrm{J}(x, \mathcal{F}) \cap E^{\prime}$ and $v \in \mathrm{J}(y, \mathcal{F}) \cap D$. As $x \in E$ and $y \in E^{\prime}$, we have $x \in \mathrm{J}(y, \mathcal{F})$ and $y \in \mathrm{J}(x, \mathcal{F})$. Hence, $x \approx_{\mathcal{F}} y$, and therefore $E=E^{\prime}$. Finally, suppose that $E \preccurlyeq E^{\prime}$ and $E^{\prime} \preccurlyeq E^{\prime \prime}$. Then $E \cap \mathrm{A}_{w u}\left(E^{\prime}\right) \neq \emptyset$ and $E^{\prime} \cap \mathrm{A}_{w u}\left(E^{\prime \prime}\right) \neq \emptyset$. Take $x \in E \cap \mathrm{A}_{w}\left(E^{\prime}\right)$ and $y \in E^{\prime} \cap \mathrm{A}_{w}\left(E^{\prime \prime}\right)$. Then there are $u \in \mathrm{J}(x, \mathcal{F}) \cap E^{\prime}$ and $v \in \mathrm{J}(y, \mathcal{F}) \cap E^{\prime \prime}$. As $y \in E^{\prime}$, it follows that $y \in \mathrm{J}(x, \mathcal{F})$ and therefore $v \in \mathrm{J}(x, \mathcal{F})$. Thus, $v \in \mathrm{J}(x, \mathcal{F}) \cap E^{\prime \prime}$, which means $E \preccurlyeq E^{\prime \prime}$.

We shall describe the maximal weak prolongational $\mathcal{F}$-control sets with respect to the dynamic order $\left(R_{\mathrm{PCS}}\right)$ in the case of $\mathcal{F}$ being a family of ideals of $S$. We need the following concept.

Definition 4.19. A subset $X \subset M$ is said to be minimal equistable if it is nonempty, closed, equistable, and has no proper subset satisfying these properties.

Proposition 4.20. Assume that $X \subset M$ is an equistable set. If $\mathrm{D}(x, S)=X$ for all $x \in X$ then $X$ is minimal equistable. 
Proof. Suppose that $Y \subset X$ is equistable. By Proposition 3.2, we have $\mathrm{D}(y, S) \subset Y$ for every $y \in Y$. Hence, $X=\mathrm{D}(y, S) \subset Y$ for any $y \in Y$, and therefore $Y=X$.

Evidently, the converse to Proposition 4.20 will be true if the prolongations are equistable sets. This fact occurs under compactness and equicontinuity.

Proposition 4.21. Assume that the action is equicontinuous. Let $\mathcal{F}$ be a family of left ideals of $S$. If the prolongational limit set $\mathrm{J}(x, \mathcal{F})$ is a nonempty compact set, then it is equistable. In particular, if $M$ is compact, then $\mathrm{J}(x, \mathcal{F})$ is a compact equistable set for all $x \in M$.

Proof. Suppose by contradiction that $\mathrm{J}(x, \mathcal{F})$ is not equistable. Then there is $y \in M \backslash \mathrm{J}(x, \mathcal{F})$ such that $y \in \operatorname{cls}(S \mathrm{~B}(\mathrm{~J}(x, \mathcal{F}), \mathcal{U}))$ for all $\mathcal{U} \in \mathcal{O}$. Hence, for each $\mathcal{U} \in \mathcal{O}$ there are $t_{\mathcal{U}} \in S, x_{\mathcal{U}} \in \mathrm{B}(\mathrm{J}(x, \mathcal{F}), \mathcal{U})$, and $z_{\mathcal{U}} \in \mathrm{J}(x, \mathcal{F})$ such that $x_{\mathcal{U}} \in \mathrm{B}\left(z_{\mathcal{U}}, \mathcal{U}\right)$ and $t_{\mathcal{U}} x_{\mathcal{U}} \in \mathrm{B}(y, \mathcal{U})$. We may assume that $\left(z_{\mathcal{U}}\right)_{\mathcal{U} \in \mathcal{O}}$ converges to a point $z \in \mathrm{J}(x, \mathcal{F})$. By Proposition 2.6, it follows that $x_{\mathcal{U}} \rightarrow z$. Since $t_{\mathcal{U}} x_{\mathcal{U}} \rightarrow y$, we have $y \in \mathrm{D}(z, S)$. As $z \in \mathrm{J}(x, \mathcal{F})$, we have $y \in \mathrm{D}(z, S)$ and $z \in \mathrm{D}(x, A)$ for every $A \in \mathcal{F}$. By Lemma 4.14, it follows that $y \in \mathrm{D}(x, A)$ for every $A \in \mathcal{F}$, because the elements of $\mathcal{F}$ are left ideals of $S$. Hence, $y \in \mathrm{J}(x, \mathcal{F})$, which is a contradiction.

In particular, in the case of equicontinuous action, the prolongation $\mathrm{D}(x, S)$ is equistable if it is compact.

Propositions 3.2 and 4.21 imply the following result.

Corollary 4.22. Assume that the action is equicontinuous and let $\mathcal{F}$ be a family of left ideals of $S$. Let $K$ be a compact equistable subset of $M$. Then $\mathrm{J}(x, \mathcal{F})$ is a compact equistable set for all $x \in K$.

The next result characterizes the compact minimal equistable sets by means of prolongational limit sets.

Proposition 4.23. Assume that the action is equicontinuous and let $\mathcal{F}$ be a family of left ideals of $S$. Then, a compact subset $K \subset M$ is minimal equistable if and only if $\mathrm{J}(x, \mathcal{F})=K$ for every $x \in K$.

Proof. If $K$ is minimal equistable and $x \in K$, Proposition 4.21 assures that $\mathrm{J}(x, \mathcal{F})$ is a compact equistable subset of $K$. The minimality of $K$ implies that $\mathrm{J}(x, \mathcal{F})=K$. As to the converse, if $\mathrm{J}(x, \mathcal{F})=K$ for all $x \in K$, then $K$ is equistable by Proposition 4.21. Now, let $K^{\prime} \subset K$ be a nonempty, closed, and equistable subset. For $x \in K^{\prime}$, we have $\mathrm{J}(x, \mathcal{F}) \subset K^{\prime}$. It follows that $K=\mathrm{J}(x, \mathcal{F}) \subset K^{\prime}$, and therefore $K=K^{\prime}$.

Although every equistable set is forward invariant, the following example shows that the concept of minimal equistable set is different from the concept of minimal set. 


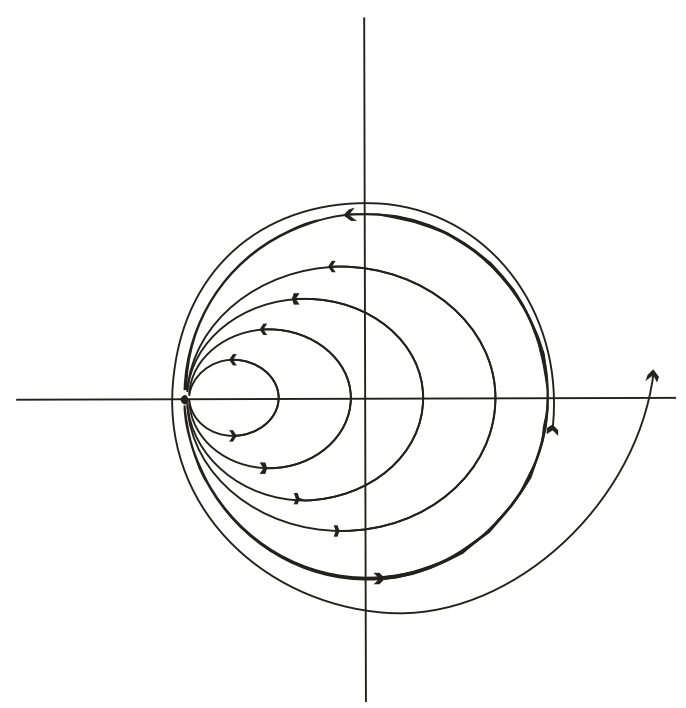

FiguRE 2. A weak prolongational control set that is not a weak control set.

ExAmple 4.24. Consider the dynamical system on $M=\mathbb{R}^{2}$ whose phase portrait is as in Figure 2. The unit circle $\mathbb{S}^{1}$ consists of a rest point $p=(-1,0)$ and a homoclinic trajectory $\Gamma$ such that $\omega(x)=\omega^{*}(x)=\{p\}$ for all $x \in \Gamma$. Hence, the unit circle is not a minimal set. However, the trajectories in the interior of $\mathbb{S}^{1}$ have the same property as $\Gamma$, and all trajectories in the exterior of $\mathbb{S}^{1}$ spiral to it as $t \rightarrow+\infty$. Hence, we have $\mathrm{J}(x)=\mathbb{S}^{1}$ for all $x \in \mathbb{S}^{1}$. By Theorem 3.3, the unit circle is a minimal equistable set.

Example 4.25. Consider the control system on $M=\mathbb{R}^{2}$ determined by the set of vector fields $F=\left\{X_{1}, X_{2}\right\}$ which have trajectories as in Figure 3 .

Let the notations be as in Example 2.4. For both vector fields, the disk $D$ is uniformly stable, the circle $C$ is a periodic trajectory (stable for $X_{1}$ and unstable for $X_{2}$ ), and the origin 0 is an equilibrium point (unstable for $X_{1}$ and stable for $X_{2}$ ). For the control system, the following statements are clear:

(1) The disk $D$ is uniformly stable.

(2) For every point $x$ outside $D, \mathrm{~J}(x, \mathcal{F})=\omega(x, \mathcal{F})=C$.

(3) For every $x \in C, \omega(x, \mathcal{F})=C$.

(4) For every $x \in \operatorname{int}(D) \backslash\{0\}, C \cup\{0\} \subset \omega(x, \mathcal{F})$.

Note that $D$ is a minimal equistable set, since there is no proper stable set inside $D$. Let $x, y \in \operatorname{int}(D) \backslash\{0\}$. For a given $T>0$, there are $t>T$ and $s>0$ such that $\mathrm{e}^{t X_{1}} x=\mathrm{e}^{-s X_{2}} y$. Hence, $\mathrm{e}^{s X_{2}} \mathrm{e}^{t X_{1}} x=y$, and therefore $y \in S_{\geq T} x$. It follows that $\operatorname{int}(D) \backslash\{0\} \subset S_{\geq T} x$ for all $x \in \operatorname{int}(D) \backslash\{0\}$ and all $T>0$. Thus, 


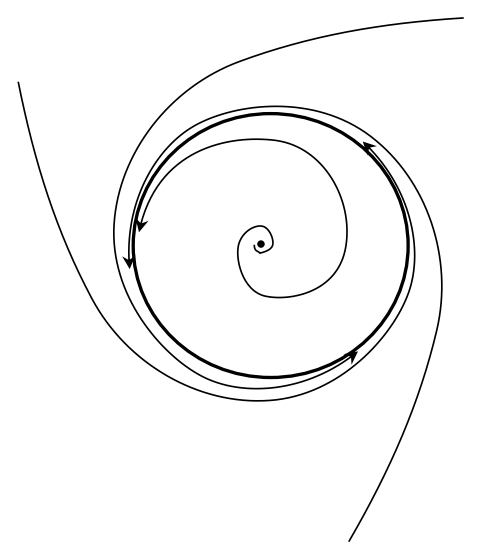

$X_{1}$

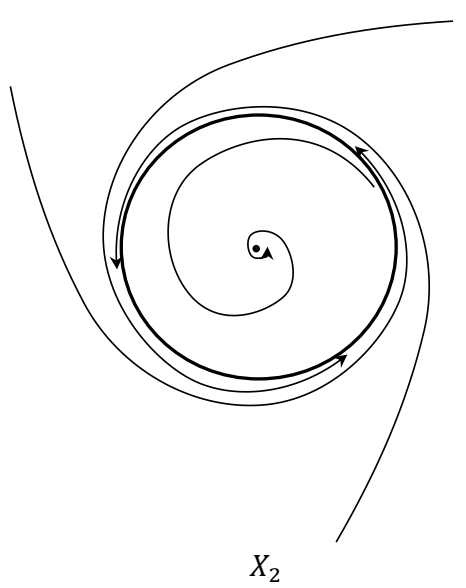

$X_{2}$

Figure 3 . Trajectories of the vector fields $X_{1}$ and $X_{2}$ in the plane.

$\operatorname{int}(D) \backslash\{0\} \subset \omega(x, \mathcal{F})$ for all $x \in \operatorname{int}(D) \backslash\{0\}$. Since $C \cup\{0\} \subset \omega(x, \mathcal{F})$, we have

$$
D \subset \omega(x, \mathcal{F}) \text { for all } x \in \operatorname{int}(D) \backslash\{0\} .
$$

Now, for $x \in C$ and $\varepsilon>0$, take $y \in \mathrm{B}(x, \varepsilon) \cap \operatorname{int}(D)$. Then $\operatorname{int}(D) \backslash\{0\} \subset$ $S_{\geq T} y \subset S_{\geq T} \mathrm{~B}(x, \varepsilon)$ for all $T>0$. As $0 \in \operatorname{cls}\left(S_{\geq T} \mathrm{~B}(x, \varepsilon)\right)$, it follows that $\operatorname{int}(D) \subset \operatorname{cls}\left(S_{\geq T} \mathrm{~B}(x, \varepsilon)\right)$ for all $\varepsilon, T>0$. Thus, $\operatorname{int}(D) \subset \mathrm{J}(x, \mathcal{F})$, and since $\omega(x, \mathcal{F})=C$, we have

$$
D \subset \mathrm{J}(x, \mathcal{F}) \text { for all } x \in C \text {. }
$$

Analogously, we have $D \subset \operatorname{cls}\left(S_{\geq T} \mathrm{~B}(0, \varepsilon)\right)$ for all $\varepsilon, T>0$, and hence $D \subset$ $\mathrm{J}(0, \mathcal{F})$. So we have $D=\mathrm{J}(x, \mathcal{F})$ for all $x \in D$, which implies that $D$ is a prolongational $\mathcal{F}$-control set for the control system.

ExAmple 4.26. An $n$-time semidynamical system is a semigroup action $(S, M)$, where $S \subset \mathbb{R}^{n}$ is a cone. Let $(S, M, \mu)$ be a two-time semidynamical system with $S=\left\{(s, t) \in \mathbb{R}^{2}: s, t \geq 0\right\}, M=\mathbb{R}^{2}$, and $\mu((s, t),(x, y))=$ $\left(e^{-s} x, e^{-t} y\right)$. Consider the family $\mathcal{F}=\left\{A_{r}: r>0\right\}$, where $A_{r}=\{(s, t) \in S$ : $s, t \geq r\}$. For any $(x, y) \in \mathbb{R}^{2} \backslash\{0\}$ and $A_{r} \in \mathcal{F}$, we have

$$
D\left((x, y), A_{r}\right)=\bigcap_{\varepsilon>0} \operatorname{cls}\left(A_{r} \mathrm{~B}((x, y), \varepsilon)\right)=\operatorname{cls}\left(A_{r}(x, y)\right)
$$

as illustrated in Figure 4. Hence, $J((x, y), \mathcal{F})=\omega((x, y), \mathcal{F})=\{0\}$, and therefore the origin 0 is weak prolongational $\mathcal{F}$-control set that is minimal equistable.

We now prove that every compact equistable set has an equistable minimal subset. We need the following lemma. 


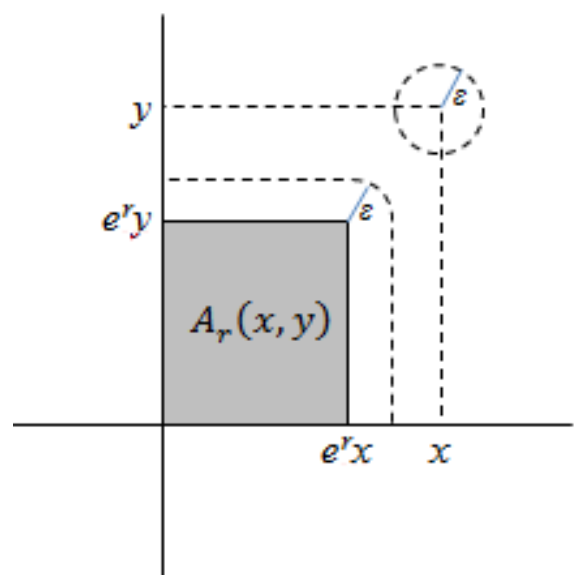

FIGURE 4. An illustration of a forward prolongation in the plane.

LEMMA 4.27. Let $\left(X_{i}\right)_{i \in I}$ be a collection of equistable subsets of $M$. Then $\bigcap_{i \in I} X_{i}$ is equistable if it is nonempty.

Proof. Take $z \notin \bigcap_{i \in I} X_{i}$. Then there is $j \in I$ such that $z \notin X_{j}$. By the equistability of $X_{j}$ there is an open covering $\mathcal{U} \in \mathcal{O}$ such that $z \notin \operatorname{cls}\left(\mathcal{S B}\left(X_{j}, \mathcal{U}\right)\right)$. Therefore $z \notin \operatorname{cls}\left(\mathcal{S B}\left(\bigcap_{i \in I} X_{i}, \mathcal{U}\right)\right)$ and $\bigcap_{i \in I} X_{i}$ is equistable.

Proposition 4.28. Every compact equistable set in $M$ has a minimal equistable subset.

Proof. Let $K \subset M$ be a compact equistable set and consider the collection $\mathfrak{C}=\{C \subset M: C$ is a closed equistable subset of $K\}$

ordered by reverse inclusion, that is, $C_{2} \geq C_{1}$ if and only if $C_{2} \subset C_{1}$. Note that $\mathfrak{C} \neq \emptyset$ because $K \in \mathfrak{C}$. Take an arbitrary chain $\left(C_{i}\right)_{i \in I}$ in $\mathfrak{C}$ and let $C=\bigcap_{i \in I} C_{i}$. The compactness of $K$ and Lemma 4.27 imply that $C$ is a nonempty upper bound of $\left(C_{i}\right)_{i \in I}$ contained in $\mathfrak{C}$. By Zorn's Lemma, we obtain a maximal element of $\mathfrak{C}$, which is a minimal equistable subset of $K$.

Finally, we are able to describe the weak prolongational $\mathcal{F}$-control sets which are maximal with respect to the order relation $\left(R_{\mathrm{PCS}}\right)$.

THEOREM 4.29. Assume that the action is equicontinuous and let $\mathcal{F}$ be a family of left ideals of $S$. If $K \subset M$ is a compact minimal equistable set, then $K$ is a weak prolongational $\mathcal{F}$-control set that is maximal with respect to the order relation $\left(R_{\mathrm{PCS}}\right)$. The converse holds if the whole space $M$ is compact. 
Proof. Suppose that $K$ is a compact minimal equistable set. By Proposition 4.23 , it follows that $\mathrm{J}(x, \mathcal{F})=K$ for all $x \in K$. Hence, there is a weak prolongational $\mathcal{F}$-control set $E$ such that $K \subset E$. For $x \in K$ and $y \in E$, we have $y \in E \subset \mathrm{J}(x, \mathcal{F})=K$. Thus, $K=E$ is a weak prolongational $\mathcal{F}$-control set. Suppose that $E^{\prime}$ is a weak prolongational $\mathcal{F}$-control set such that $K \preccurlyeq E^{\prime}$. Then there is $x \in K \cap \mathrm{A}_{w u}(E, \mathcal{F})$, that is, $\mathrm{J}(x, \mathcal{F}) \cap E \neq \emptyset$. As $\mathrm{J}(x, \mathcal{F})=K$, it follows that $K=E^{\prime}$. Thus, $K$ is maximal with respect to $\left(R_{\mathrm{PCS}}\right)$. Now, suppose that $M$ is compact and $K$ is a weak prolongational $\mathcal{F}$-control set that is maximal with respect to $\left(R_{\mathrm{PCS}}\right)$. For $x \in K, \mathrm{~J}(x, \mathcal{F})$ is compact and equistable. By Proposition 4.28, there is a minimal equistable set $K^{\prime}$ contained in $\mathrm{J}(x, \mathcal{F})$. By the first part of the proof, $K^{\prime}$ is a weak prolongational $\mathcal{F}$-control set. Since $x \in K \cap \mathrm{A}_{w u}\left(K^{\prime}, \mathcal{F}\right)$, we have $K \preccurlyeq K^{\prime}$. By the maximallity of $K$, it follows that $K=K^{\prime}$. Therefore, $K$ is minimal equistable.

\section{Conclusion}

We have seen that forward (backward) invariant compact sets are weakly forward (backward) $\mathcal{F}$-Poisson stable, and invariant compact sets are $\mathcal{F}$-Poisson stable (Proposition 3.12). As a consequence, compact Lyapunov stable or equistable sets are weakly forward $\mathcal{F}$-Poisson stable. Moreover, if $X$ is a Lagrange stable set, then $\operatorname{cls}(S X)$ is weakly forward $\mathcal{F}$-Poisson stable. If the family $\mathcal{F}$ satisfies hypothesis $\left(\mathrm{H}_{1}\right)$, then the weak $\mathcal{F}$-control sets coincide with the equivalence classes of the equivalence relation in the set of all $\mathcal{F}$-Poisson stable points. In this case, weak $\mathcal{F}$-control sets are $\mathcal{F}$-Poisson stable. On the other hand, every forward $\mathcal{F}$-Poisson stable point is contained in a weak $\mathcal{F}$-control set (Proposition 4.8). Although the controllability is totally different from the Lyapunov stability, we have discovered a situation in what they are connected. We have introduced the notions of weak prolongational control set and minimal equistable set. If $\mathcal{F}$ is a family of left ideals of the semigroup $S$ and the action of $S$ on the space $M$ is equicontinuous, then a compact minimal equistable set is a weak prolongational $\mathcal{F}$-control set that is maximal with respect to the dynamic order among the weak prolongational $\mathcal{F}$-control sets, and the converse holds if the whole space $M$ is compact (Theorem 4.29).

\section{REFERENCES}

[1] N.P. Bhatia And O. HajeK, Local semi-dynamical systems, Lecture Notes in Mathematics 90, Springer-Verlag, 1969.

[2] N.P. Bhatia And G.P. Szegő, Dynamical systems: stability theory and applications, Lecture Notes in Mathematics 35, Springer-Verlag, 1967.

[3] _ Stability theory of Dynamical Systems, Springer-Verlag, 1970.

[4] C.J. Braga Barros and L.A.B. San Martin, On the action of semigroups in fiber bundles, Mat. Contemp. 13 (1997), 1-19. 
[5] C.J. Braga Barros AND J.A. SouzA, Attractors and chain recurrence for semigroup actions, J. Dynam. Differential Equations 22 (2010), 723-740.

[6] C.J. Braga Barros, V.H.L. Rocha And J.A. Souza, Lyapunov stability for semigroup actions, Semigroup Forum 88 (2014), 227-249.

[7] M. Patrão L.A.B. San Martin, Morse decomposition of semiflows on fiber bundles, Discrete Contin. Dyn. Syst. 17 (2007), 113-139.

[8] _ Semiflows on topological spaces: chain transitivity and semigroups, J. Dynam. Diferential Equations 19 (2007), 155-180.

[9] S.A. Raminelli and J.A. SouzA, Global attractors for semigroup actions, J. Math. Anal. Appl. 407 (2013), 316-327.

[10] L.A.B. SAn Martin, Invariant control sets on flag manifolds, Math. Control Signals Systems 6 (1993), 41-61.

[11] _ Control sets and semigroups in semi-simple Lie groups, Semigroups in Algebra, Geometry and Analysis, Gruyter Verlag, 1994.

[12] L.A.B. San Martin And P.A. Tonelli, Semigroup actions on homogeneous spaces, Semigroup Forum 50 (1995), 59-88.

[13] J.A. SouzA, Complete Lyapunov functions of control systems, Systems Control Lett. 61 (2012), 322-326.

[14] _ Lebesgue covering lemma on nonmetric spaces, Internat. J. Math. 24 (2013), 1350018, 1-12.

[15] _ On limit behavior of semigroup actions on noncompact space, Proc. Amer. Math. Soc. 140 (2012), 3959-3972.

[16] _ On limit behavior of skew-product transformation semigroups, Math. Nachr. 287 (2014), 91-104.

[17] _ Recurrence theorem for semigroup actions, Semigroup Forum 83 (2011), 351-370.

[18] J.A. Souza And H.V.M. Tozatti, Prolongational limit sets of control systems, J. Differential Equations 254 (2013), 2183-2195.

[19] _ Some aspect of stability for semigroup actions, J. Dynam. Differential Equations 26 (2014), 631-654.

Manuscript received December 5, 2014

accepted July 2, 2015

Josiney A. Souza And Victor H.L. Rocha

Departamento de Matemática

Universidade Estadual de Maringá

Maringá, BRASIL

E-mail address: jasouza3@uem.br

HÉlio V.M. TozATti

Departamento de Matemática

Universidade Tecnológica Federal do Paraná

Campo Mourão, BRASIL 